\title{
Disorders of Riboflavin Metabolism
}

Shanti Balasubramaniam ${ }^{1,2,3,4}$ John Christodoulou ${ }^{3,4,5,6}$, Shamima Rahman ${ }^{7,8}$

${ }^{1}$ Western Sydney Genetics Program, The Children's Hospital at Westmead, Sydney, NSW, Australia

${ }^{2}$ Kids Research Institute, The Children's Hospital at Westmead, Sydney, NSW, Australia

${ }^{3}$ Discipline of Genetic Medicine, Sydney Medical School, University of Sydney, Sydney, NSW, Australia

${ }^{4}$ Discipline of Child \& Adolescent Health, Sydney Medical School, University of Sydney, Sydney, NSW, Australia

${ }^{5}$ Neurodevelopmental Genomics Research Group, Murdoch Children's Research Institute, Melbourne, VIC, Australia

${ }^{6}$ Department of Paediatrics, Melbourne Medical School, University of Melbourne, Melbourne, VIC, Australia

${ }^{7}$ Mitochondrial Research Group, UCL Great Ormond Street Institute of Child Health, London, UK

${ }^{8}$ Metabolic Unit, Great Ormond Street Hospital NHS Foundation Trust, London, UK

\section{Corresponding author:}

Professor Shamima Rahman

Mitochondrial Research Group,

UCL Great Ormond Street Institute of Child Health,

This is the author manuscript accepted for publication and has undergone full peer review but has not been through the copyediting, typesetting, pagination and proofreading process, which may lead to differences between this version and the Version of Record. Please cite this article as doi: $10.1002 /$ jimd.12058

This article is protected by copyright. All rights reserved. 
London, United Kingdom

Phone: $+44(0) 2079052608 \quad$ Fax: $+44(0) 2074046191$

E-mail: shamima.rahman@ucl.ac.uk

\begin{abstract}
Riboflavin (vitamin B2), a water-soluble vitamin, is an essential nutrient in higher organisms as it is not endogenously synthesized, with requirements being met principally by dietary intake. Tissue-specific transporter proteins direct riboflavin to the intracellular machinery responsible for the biosynthesis of the flavocoenzymes flavin mononucleotide (FMN) and flavin adenine dinucleotide (FAD). These flavocoenzymes play a vital role in ensuring the functionality of a multitude of flavoproteins involved in bioenergetics, redox homeostasis, DNA repair, chromatin remodelling, protein folding, apoptosis and other physiologically relevant processes. Hence, it is not surprising that the impairment of flavin homeostasis in humans may lead to multisystem dysfunction including neuromuscular disorders, anaemia, abnormal fetal development and cardiovascular disease. In this review we provide an overview of riboflavin absorption, transport, and metabolism. We then focus on the clinical and biochemical features associated with biallelic FLAD1 mutations leading to FAD synthase deficiency, the only known primary defect in flavocoenzyme synthesis, in addition to providing an overview of clinical disorders associated with nutritional deficiency of riboflavin and primary defects of riboflavin transport. Finally, we give a brief overview of disorders of the cellular flavoproteome. Because riboflavin therapy may be beneficial in a number of primary or secondary disorders of the cellular flavoproteome, early recognition and prompt management of these disorders is imperative.
\end{abstract}




\section{Keywords:}

Riboflavin metabolism, riboflavin transport, flavocoenzyme, flavoprotein, flavoproteome, FAD synthase deficiency, FLAD1 mutations, riboflavin responsive disorders.

This article is protected by copyright. All rights reserved. 


\section{Key messages:}

- Riboflavin is an essential nutrient in higher organisms since it is not endogenously synthesized and must be supplied through dietary intake.

- Primary defects of flavocoenzyme metabolism include genetically inherited disorders: a) involving enzymes in the synthetic pathway of essential cofactors FMN and FAD (i.e. FAD synthase deficiency; to date, no cases of human riboflavin kinase deficiency have been reported).

b) disorders of riboflavin or flavocoenzyme transport.

- Secondary flavocoenzyme defects refer to the functional disruption of the cellular flavoproteome, i.e. cofactor dependent reactions which do not arise as a direct result of defects in the synthesis or transport of cofactors. Examples include multiple acylCoA dehydrogenase deficiency (MADD), glutaric aciduria type 1, and dihydrolipoamide dehydrogenase deficiency.

- Both primary and secondary flavocoenzyme deficiencies may present with abnormal biochemical features including characteristic elevations of plasma acylcarnitines and urinary organic acids.

- Riboflavin therapy may be beneficial in both primary and secondary flavocoenzyme deficiencies, hence early recognition of these disorders is imperative.

\section{Details of the Contributions of Individual Authors}

All three authors contributed to writing and critically revising the manuscript. 


\section{Introduction}

Riboflavin (7,8-dimethyl-10-ribityl-isoalloxazine), also known as vitamin B2, plays a role in a wide range of metabolic pathways, because it is a precursor of the essential cofactors flavin mononucleotide (FMN) and flavin adenine dinucleotide (FAD) which are utilised in numerous enzymatic reactions. The human flavoprotein has a clear bias towards FADdependent enzymes which account for $84 \%$ of human flavoproteins, whilst only $16 \%$ use FMN as a cofactor (Joosten 2007, Lienhart et al 2013). Flavocoenzymes are able to catalyse one-electron and two-electron transfer reactions in oxidation-reduction processes, making them key players in a number of critical metabolic processes including the mitochondrial electron transport chain, mitochondrial and peroxisomal fatty acid $^{2}$-oxidation, the citric acid cycle, nitric oxide synthases, redox homeostasis and branched-chain amino acid catabolism. These flavoproteins are also important in chromatin remodelling, DNA repair, protein folding and apoptosis (Joosten et al 2007). Finally, flavoenzymes also play an important role in the biosynthesis or regulation of other essential cofactors and hormones, such as coenzyme A, coenzyme $\mathrm{Q}_{10}$, haem, steroids and thyroxine, as well as metabolism of other $\mathrm{B}$ vitamins (folate, pyridoxine/pyridoxal 5'-phosphate and niacin) (Lienhart et al 2013;Kennedy 2016).

\section{Dietary sources of riboflavin}


Riboflavin, as with other water-soluble B vitamins, must be obtained through the diet, since higher organisms have lost the ability to synthesize this molecule as opposed to various plants, fungi and bacteria. Endogenous synthesis of riboflavin (from ribulose-5-phosphate and GTP) by bacteria in the large intestine may contribute to some extent (Barile et al 2016). The recommended daily allowance (RDA) of riboflavin on average is $1.3 \mathrm{mg} /$ day for adult men and $1.1 \mathrm{mg} /$ day for women, with variations depending on age and reproductive status including pregnancy and lactation (Barile et al 2016). Milk and dairy products are the largest sources of riboflavin in the Western diet, making up 51\% of intake in preschool children, $35 \%$ in school children, $27 \%$ in adults, and $36 \%$ in the elderly (Powers 2003). Due to the adequate availability of riboflavin in a wide variety of food sources, isolated deficiency is not prevalent in the general population, apart from rare genetic defects and malabsorptive conditions (Pinto and Zempleni 2016).

\section{Riboflavin transport and metabolism}

Riboflavin ingested in the diet exists either as free riboflavin or its protein bound form as flavoproteins containing FAD and FMN. Milk and eggs contain free riboflavin, but most other foods tend to predominantly contain FAD and FMN, which must be released from the carrier proteins to which they are bound. This is achieved by protein denaturation in the stomach, and subsequent hydrolysis to free riboflavin by alkaline phosphatases and FMN/FAD pyrophosphatases in the brush border of the ileal enterocyte, allowing absorption in the small intestine (Henriques et al 2013). 
Three human plasma membrane riboflavin transporters have been characterised (Figure 1a): RFVT1 (previously hRFT1), RFVT2 (hRFT3) and RFVT3 (hRFT2), encoded by SLC52A1, SLC52A2 and SLC52A3 respectively (Yonezawa and Inui 2013). These three transporters have different subcellular locations and tissue specificities (Yonezawa and Inui 2013; Barile et al 2016). Free riboflavin is transported into enterocytes via carrier-mediated uptake by RFVT3 (Yonezawa and Inui 2013), a saturable transport process at the apical membrane which is reported to be linear up to approximately $30 \mathrm{mg}$ riboflavin per meal (Levy 1966; McCormick 1989; Powers 2003), and beyond which little additional absorption of riboflavin occurs (Zempleni et al 1996; Powers 2003).

After cellular uptake, riboflavin is quickly transformed into its catalytically active cofactors by the action of two enzymes: riboflavin kinase (RFK) (EC 2.7.1.26) and FAD synthase (FADS) (EC 2.7.7.2) which catalyse FMN and FAD production respectively (Figure 1b). Riboflavin may be released into the portal blood and to the liver in its free form or as FMN after being transported by RFTV1 and RFVT2 which are embedded within the basolateral membrane of enterocytes (Barile et al 2016). Circulating plasma riboflavin is bound to either albumin or immunoglobulins or is converted into its coenzyme forms in erythrocytes or leukocytes. Median plasma concentrations for riboflavin, FMN and FAD have been reported to be 10.5, 6.6 and $74 \mathrm{nmol} / \mathrm{L}$ (Hustad et al 2002; Barile 2016).

- RFK is a ubiquitous rate-limiting flavokinase that catalyses the first step in FAD biosynthesis by phosphorylating riboflavin to form FMN (Karthikeyan et al 2003), which is 
subsequently adenylated by FADS to generate FAD (Brizio et al 2006). The crystal structure of human RFK revealed a six-stranded antiparallel beta-barrel core that complexes with both riboflavin and ADP (Karthikeyan et al 2003). RFK has also been shown to be a tumour necrosis factor (TNF) receptor-1 (TNFR1)-binding protein that physically and functionally couples TNFR1 to NADPH oxidase. TNF, through the activation of RFK enhances the incorporation of FAD into NADPH oxidase, which is critical for the assembly and activation of NADPH oxidase (Yazdanpanah et al 2009).

FADS catalyses the adenylation of FMN to FAD (Brizio et al 2006). In humans, distinct isoforms of this enzyme are distributed in different subcellular compartments and generated by alternative splicing of the FLAD1 gene (Torchetti et al 2010; Giancaspero et al 2013; Barile et al 2016; Olsen et al 2016). The most abundant of these isoforms, i.e. the cytosolic enzyme or isoform 2, has been shown to be a bifunctional enzyme with both FAD synthase and hydrolase activity (Giancaspero et al 2015). The enzyme contains an N-terminal molybdopterin binding (MPTb) domain which has FAD hydrolase activity, and a C-terminal 3'-phosphoadenosine-5' -phosphosulphate (PAPS) reductase domain, which is sufficient to catalyse FAD synthesis and so has been renamed the FADS domain (Giancaspero et al 2015; Olsen et al 2016). Very recently, a novel FADS6 isoform containing only the PAPS reductase domain was discovered in patients harbouring frameshift mutations of FLADl (Leone et al 2018). Functional characterisation suggested that FADS6 may represent an "emergency" enzyme that provides cytosolic FAD synthesizing capacity in the presence of biallelic FLAD1 frameshift variants, thus allowing survival of affected patients. The FAD synthesizing 
activity of FADS6 is approximately 70\% that of human FADS2 (Torchetti et al 2011); however, its catalytic efficiency $(\mathrm{kcat} / \mathrm{Km})$ is higher due to a lower FADS6 $\mathrm{Km}$ for both FMN and ATP compared to FADS2. FADS6 is expected to be only cytosolic since it lacks the mitochondrial targeting peptide, and might regulate the mitochondrial FAD transporter direction of transport by ensuring FAD import to facilitate flavoprotein biogenesis (Leone et al 2018).

SLC25A32 is an inner mitochondrial membrane carrier that imports FAD from the cytosol into mitochondria (mitochondrial FAD- transporter; Figure 1b), where it is needed for the many FAD-dependent mitochondrial enzymes, including components of the respiratory chain and the fatty acid beta oxidation spiral. SLC25A32 was initially identified as a mitochondrial folate transporter (Titus and Moran 2000), before it was demonstrated to be the human orthologue of the yeast mitochondrial FAD transporter FLX1 (Spaan et al 2005). The mitochondrial translocator FLX1 catalyses the bidirectional movement of the redox cofactor FAD across the mitochondrial membrane (Giancaspero et al 2014). The possibility that SLC25A32 regulates FAD efflux from the mitochondrial matrix to the cytosol has been neither confirmed nor excluded in human cells.

Unbound flavins are rapidly hydrolysed to free riboflavin and excreted in urine. Riboflavin is not stored in the body, hence any intake in excess of tissue requirements or which surpasses renal reabsorption, is eliminated in the urine as riboflavin or its catabolites 7-alpha-hydroxy riboflavin, 10-hydroxyethylflavin and lumiflavin (Chastain and McCormick 1987; Barile 
2016). This means that riboflavin has a relatively low toxicity even at supra-pharmacological doses (Barile et al 2016).

\section{Riboflavin disorders in humans}

\subsection{Nutritional riboflavin deficiency}

Since riboflavin is not endogenously synthesized or stored in human tissues, adequate dietary intake is imperative. Clinical problems associated with dietary deficiency of riboflavin, also known as ariboflavinosis, include night blindness, cataracts, fatigue, anaemia, growth retardation, migraines, peripheral neuropathy and dermatological symptoms (cheilosis, glossitis, scaly skin rashes confined to the scrotum and vulva, and chapped lips) (Smith and Garg 2017). Low riboflavin intake during pregnancy may be associated with an increased risk of congenital heart disease (Smedts et al 2008). Symptomatic riboflavin deficiency often occurs in association with deficiencies of other vitamins and has usually been attributed to dietary restriction, however it is potentially more widespread than currently recognised with an estimated $10-15 \%$ of the global population having an inherited restriction in their ability to absorb or utilise riboflavin (Kennedy 2016; Marashly et al 2017). Supplementation for prevention of ariboflavinosis is generally not necessary as adequate amounts of riboflavin are available in a healthy diet, except when the diet is very limited or other health issues are present. Riboflavin deficiency can however occur during lactation, phototherapy in infants, or as a consequence of coeliac disease, alcoholism, malignancies, or use of prescription drugs 
including phenothiazine-derived antipsychotic medications, the antimalarial drug quinacrine, phenobarbitol and the cancer chemotherapy agent adriamycin (Buehler 2011).

\subsection{Disorders of riboflavin transport}

\subsubsection{Brown-Vialetto-Van Laere syndrome}

Deficiency of the plasma membrane riboflavin transporters SLC52A2 (OMIM \#614707) and SLC52A3 (OMIM \#211530) are associated with genetic neuronopathies. These include type 1 riboflavin transporter deficiency neuronopathy caused by mutations in the $S L C 52 A 3$ gene, also known as Brown-Vialetto-Van Laere syndrome-1 (BVVLS1), a progressive neurologic disorder with bulbar palsy and sensorineural deafness (Green et al 2010), or Fazio-Londe disease, a disorder similar to BVVLS1 but without sensorineural deafness (Bosch et al 2011). Type 2 riboflavin transporter deficiency neuronopathy, or Brown-Vialetto-Van Laere syndrome-2 (BVVLS2) is caused by mutations in SLC52A2. Haploinsufficiency of the SLC52A1 riboflavin transporter (OMIM \#615026) has been reported in two neonates, associated with maternal riboflavin deficiency, who developed transient severe symptoms resembling multiple acyl-CoA dehydrogenase deficiency (MADD), which resolved with oral supplementation of riboflavin (Ho et al 2011, Mosegaard et al 2017). Disorders of riboflavin transport are discussed in detail in the accompanying review in this issue of the Journal of Inherited Metabolic Disease (O’Callaghan et al 2019).

\subsubsection{Mitochondrial FAD transporter deficiency (OMIM \# 616839)}


Two patients with biallelic mutations in SLC25A32 encoding the mitochondrial FAD transporter have been reported (Schiff et al 2016, Hellebrekers et al 2017). The first was a 14year-old girl with riboflavin-responsive recurrent exercise intolerance and biochemical features of MADD (Schiff et al 2016). The second patient, 51 years old at the time of reporting, presented at 3 years with muscle weakness following an episode of influenza, and subsequently had progressive exercise intolerance in childhood, together with neurological symptoms including early-onset ataxia, myoclonus, dysarthria and dysphagia (Hellebrekers et al 2017). Muscle biopsy revealed ragged-red fibres, lipid storage and fibres with decreased staining for succinate dehydrogenase (SDH, FAD-dependent mitochondrial respiratory chain complex II) and cytochrome $c$ oxidase (COX, mitochondrial respiratory chain complex IV) in both patients. Deficiency of complex II was demonstrated in muscle from the second patient and cultured skin fibroblasts from the first patient (Hellebrekers et al 2017, Schiff et al 2016). Both patients had dramatic improvements in the clinical and biochemical abnormalities following oral riboflavin supplementation, including improved exercise tolerance and endurance (Schiff et al 2016, Hellebrekers et al 2017).

It is interesting that these patients have a MADD-like predominantly myopathic presentation, quite distinct from the neuropathic presentation of patients with mutations in the plasma membrane riboflavin transporters RFVT2 and RFVT3.

\subsection{Primary defect of riboflavin coenzyme metabolism}

\subsubsection{FAD synthase deficiency (OMIM \# 255100)}

In the last few years biallelic variants in FLADI encoding FADS have been identified as 
causing a potentially treatable neuromuscular disease manifesting with lipid storage myopathy and metabolic abnormalities suggestive of MADD, in association with multiple respiratory chain enzyme deficiencies (Taylor et al 2014, Olsen et al 2016). Thirteen patients have been reported in the literature to date, all but one patient (Ryder et al 2018) were identified by whole exome sequencing or through candidate gene panels, and their clinical features are summarised in Table 1 (Taylor et al 2014, Olsen et al 2016, Auranen et al 2017, Yildiz et al 2018). Onset was in infancy in eleven cases (85\%). Presenting features in almost all of these infants included hypotonia and severe muscle weakness leading to feeding difficulties (poor suck and swallow, frequently necessitating tube feeding) and respiratory insufficiency. One patient presented with cardiorespiratory collapse at 32 hours of age and died at 3 days (Olsen et al 2016). Another had cardiomyopathy in the first year of life and recurrent supraventricular tachycardias necessitating implantable cardioverter defibrillator placement. This infant had a dramatic response to riboflavin supplementation and was still alive at 22 years (Olsen et al 2016). Another infant who needed a pacemaker because of recurrent episodes of sudden cardiac arrest died of multiorgan failure at 7 months (Olsen et al 2016). Eight of the eleven cases with an infantile onset died, seven within the first 9 months of life and the eighth at 16 years (Taylor et al 2014, Olsen et al 2016, Yildiz et al 2018). Three children who presented in infancy were alive, two at 8 years and the third at 22 years, all treated with riboflavin. Two individuals presented in adult life, at 20 and 44 years, and were alive at 44 and 56 years respectively (Olsen et al 2016, Auranen et al 2017). The adultonset cases presented with exercise intolerance and progressive muscle weakness, with gait 
difficulties, bilateral foot drop and arm weakness in the older patient (Olsen et al 2016, Auranen et al 2017).

Blood and urine biochemistry in FADS deficiency are typical of riboflavin deficiency or MADD, with increased acylcarnitines and urinary organic acids in all cases tested (Table 2). Elevations of C4-C14 even chain acylcarnitines, C5 and C5-DC and C10:1, C14:1, C16:1, C18:1 and C18:2 have all been reported (Olsen et al 2016, Yildiz et al 2018). In one infant only C4 was elevated, and in another the acylcarnitine profile was normal on some occasions and abnormal on others (Olsen et al 2016). Increased urinary excretion of ethylmalonic, adipic, suberic, glutaric and methylsuccinic acids, hexanoylglycine and tiglylglycine has been observed (Olsen et al 2016, Yildiz et al 2018, Ryder et al 2018). There appears to be a characteristic muscle pathology in FADS deficiency, with pronounced lipid storage and a global decrease of COX and/or SDH histochemical staining (Taylor et al 2014). Ragged-red fibres have not been reported so far. Multiple respiratory chain enzyme deficiencies were observed in skeletal muscle biopsies in 7 of 8 cases tested, variably affecting complexes I, II, III and/or IV (Taylor et al 2014; Olsen et al 2016; Yildiz et al 2018). One case was said to have normal muscle respiratory chain enzyme activities (Olsen et al 2016). Decreased FADS activity was demonstrated in cultured skin fibroblasts from all four patients tested (Olsen et al 2016, Ryder et al 2018).

Nine of the 13 patients reported with FADS deficiency were Turkish, and three different homozygous FLAD1 mutations were observed in this population (Table 1). One mutation 
appears to be particularly prevalent, suggesting a possible founder effect. This is a $4 \mathrm{bp}$ deletion (c.401_404delTTCT, initially reported as c.397_400delTTCT) in the MPTb domain resulting in a frameshift and premature termination (p.(Phe134CysfsTer8)), and was observed in five infants from four families (Taylor et al 2014, Olsen et al 2016, Yildiz et al 2018). All five died between 4 and 8 months of age. A possible genotype-phenotype correlation has been suggested (Olsen et al 2016). Three of four individuals with at least one FADS domain mutation affecting a single amino acid had prolonged survival (alive at 22-56 years), although the fourth died at only 3 days of life. Conversely, all 8 patients with biallelic frameshift mutations in the MPTb domain died, almost all in infancy (Table 1). One individual diagnosed with MADD on newborn screening was treated with riboflavin for the first 29 months of life, but developed a progressive myopathy from 3 years of age and was found to have a homozygous nonsense mutation in the MPTb domain of FLAD1 at 8 years (Ryder et al 2018). The nonsense mutation led to a truncated protein with some residual FADS activity (Ryder et al 2018). Clearly only a small number of patients with FLAD1 mutations have been identified so far, and so it is difficult to make firm genotype-phenotype predictions at present.

Riboflavin supplementation resulted in clinical improvements in seven of eight patients treated (Olsen et al 2016, Yildiz et al 2018, Ryder et al 2018), including dramatic amelioration of muscle symptoms and an increase in muscle strength, and improvements of the biochemical abnormalities in one adult patient who was further described in a subsequent report. This individual also received dietary therapy with high carbohydrate, moderate protein and low fat (max 20g fat/day), which was reported to ameliorate her symptoms (Auranen et al 
2017). Treatment with riboflavin at age three months in two patients homozygous for the c.401_404delTTCT mutation in FLAD1 seemed to result in mild improvements in spontaneous activity, muscle tone, vomiting and alertness, but failed to prevent disease progression; both infants died before the age of 6 months (Yildiz et al 2018). It has been proposed that biallelic frameshift mutations in exon 2 of FLAD1 may be partially responsive to riboflavin supplementation (Olsen et al 2016), but the doses used so far have not led to long-term survival (Yildiz et al 2018). Studies of the overexpressed mutant proteins in Escherichia coli demonstrated that FAD supplementation significantly improved protein stability and /or folding of the mutated proteins, supporting a chaperone-like action of FAD (Olsen et al 2016). Of note, riboflavin treatment initiated in the newborn period may have attenuated an otherwise severe phenotype in an 8-year-old boy with a homozygous nonsense mutation in FLADI who initially presented with MADD biochemical abnormalities in the newborn screening test (Ryder et al 2018).

In summary, all patients with FADS deficiency should receive high-dose riboflavin therapy, and if the diagnosis is suspected riboflavin supplementation should be implemented whilst awaiting mutational analysis, with subsequent withdrawal if the diagnosis is not confirmed. The benefits of early diagnosis and treatment are exemplified by discordant outcome between treated and untreated siblings (Olsen et al 2016). The potential avenue of prenatal or presymptomatic postnatal treatment with high dose riboflavin that may prevent or ameliorate the disease symptoms has been raised but requires further exploration. Other future therapeutic strategies include enhancing the activity of the truncated FADS isoform 6 (Leone 
et al 2018) or the mitochondrial FAD transporter in those patients who do not benefit from riboflavin (Yildiz et al 2018).

\subsubsection{Riboflavin kinase deficiency}

RFK deficiency has never been reported in humans. It is possible that this might be incompatible with life since, as discussed above, RFK is the rate-limiting step in the synthesis of FAD, an essential cofactor for many cellular reactions. In support of this hypothesis, RFK deficiency was embryonic lethal before day 7.5 of gestation in a knockout mouse model (Yazdanpanah et al 2009). However, it is possible that hypomorphic $R F K$ mutations may result in a clinical presentation similar to that seen in patients with FLADI mutations. Whole exome and whole genome sequencing of undiagnosed patients with lipid storage myopathies and MADD-like clinical presentations may identify affected cases in the fullness of time.

\subsection{Disorders of the human flavoproteome}

The human flavoproteome has been reported to comprise at least 90 distinct proteins, with diverse roles as indicated above (also see Table 3) (Lienhart et al 2013). Human diseases have been associated with approximately half of these proteins and are clinically extremely heterogeneous, including Leigh syndrome and other mitochondrial respiratory chain disorders, mitochondrial and peroxisomal fatty acid oxidation disorders, pyridoxal phosphate responsive epilepsy, trimethylaminuria, rhizomelic chondrodysplasia punctata, porphyria variegata, chronic granulomatous disease, and defects of steroidogenesis and bile acid synthesis (Table 3). Riboflavin responsiveness has not been reported for many of these 
disorders, and for several conditions it is not clear whether therapeutic trials of pharmacological doses of riboflavin have been explored as potential treatments. A full description of all disorders of the human flavoproteome is beyond the scope of this review, but some of the mitochondrial disorders associated with riboflavin responsiveness are discussed briefly below.

MADD (OMIM \#231680) is caused by deficiency of electron-transfer flavoprotein dehydrogenase (encoded by ETFDH) or of one of the two electron-transfer flavoproteins (encoded by ETFA and $E T F B$ ) which transfer electrons from acyl-CoA dehydrogenases to coenzyme $\mathrm{Q}_{10}$ in the mitochondrial respiratory chain (Olsen et al 2003). Clinical phenotypes associated with MADD are heterogeneous and have been classified into a severe neonatal onset multsystemic form and a milder later onset myopathic form. The latter is the most frequent clinical presentation, manifesting as exercise intolerance, occasionally respiratory insufficiency or rhabdomyolysis and is typically riboflavin responsive (Olsen et al 2007; Grünert 2014). Other cases may have a secondary coenzyme $\mathrm{Q}_{10}$ deficiency (Gempel et al 2007). Riboflavin supplementation has successfully ameliorated clinical symptoms and metabolic abnormalities in over $95 \%$ of patients with late-onset MADD patients, the majority of whom (93\%) have ETFDH mutations (Grünert 2014).

ACAD9 is a bifunctional enzyme primarily involved in complex I biogenesis, with an additional moonlighting function in fatty acid oxidation (FAO) (Nouws et al 2014). The major clinical presentations of ACAD9 deficiency are early-onset cardiac involvement 
ranging from isolated electrical hypertrophy to severe hypertrophic, dilated or combined cardiomyopathy, exercise intolerance and myopathy, lactic acidosis and neurological abnormalities including stroke-like episodes, ataxic gait, bradykinesia and bradylalia (Dewulf et al 2016). $A C A D 9$ mutations are the most frequent cause of hypertrophic cardiomyopathy with isolated complex I deficiency (Collet et al 2016). Riboflavin has been shown to directly increase ACAD9 protein levels and rescue complex I assembly and may also function as a chemical chaperone by improving the folding of specific ACAD9 mutant proteins (Nouws et al 2014). Therapeutic response to riboflavin supplementation was reported in $65 \%$ of treated patients in one large multinational cohort of ACAD9 deficiency (Repp et al 2018). Riboflavin responsiveness has also been reported in other forms of complex I deficiency (Ogle et al 1997). It is possible that FMN may stabilise the complex I holoenzyme.

Dihydrolipoamide dehydrogenase (also known as E3, encoded by $D L D$ ) is a flavoprotein shared by three mitochondrial \pm -ketoacid dehydrogenase multi-enzyme complexes: pyruvate dehydrogenase complex (PDHc), \pm -ketoglutarate dehydrogenase complex (KGDC) and branched-chain \pm -keto acid dehydrogenase complex (BCKDC). The metabolic derangements reflect PDHC and TCA cycle defects, and include increased blood lactate and pyruvate, elevated plasma alanine, branched-chain amino acids and increased urinary lactic, pyruvic, 2ketoglutaric, and branched-chain 2-hydroxy- and 2-ketoacid (De Meirleir et al 2016). The phenotypic spectrum of DLD deficiency is variable, and encompasses early onset encephalopathy with progressive hypotonia, failure to thrive, hypoglycemia, ketoacidosis and encephalopathy, Leigh syndrome, and a recurrent Reye-like presentation with normal intellect 
and no residual neurologic deficits between acute metabolic episodes (Quinonez et el 2014). A riboflavin-responsive myopathic phenotype with exertional fatigue, intermittent elevation of blood lactate, ketoacidosis, elevation of creatine kinase and mitochondrial proliferation has also been described (Carrozzo et al 2014). Riboflavin supplementation led to complete resolution of muscle weakness, improvement of metabolic abnormalities, partial restoration of the DLD protein, and reduced ROS production in fibroblasts, supporting a chaperone-like effect of riboflavin promoting DLD protein stability and folding (Carrozzo et al 2014).

The X-linked AIFMI gene encodes apoptosis-inducing factor (AIF), a mitochondrial flavoprotein with a dual role as an FAD-dependent NADH oxidoreductase involved in oxidative phosphorylation and redox control and also as a caspase-independent death effector mediating programmed cell death when translocating from mitochondria to the nucleus on exposure to apoptotic stimuli (Joza et al 2009). AIFM1 mutations have been associated with a wide spectrum of clinical phenotypes ranging from a severe, early-onset mitochondrial encephalomyopathy with combined oxidative phosphorylation deficiency (OMIM \#300816) (Ghezzi et al 2010), to Cowchock syndrome, an X-linked Charcot-Marie-Tooth disease with axonal sensorimotor neuropathy, sensorineural deafness and cognitive impairment (CMTX4, OMIM \#310490) (Rinaldi et al 2012). Recently AIFM1 mutations have been reported to cause cerebellar ataxia partially responsive to riboflavin (Heimer et al 2018).

\section{Conclusions}


There is increasing evidence for a potential role of riboflavin in the prevention and treatment of various primary or secondary defects of maintenance of the cellular flavoproteome. In recent years, defects of FAD synthase in the flavocoenzyme biosynthetic pathway, the plasma membrane riboflavin transporters and the mitochondrial FAD transporter have all been linked to human disease. Human riboflavin kinase deficiency remains to be described. It is not known why the plasma membrane riboflavin transporter disorders manifest predominantly as a neuronopathy (BVVL) whereas defects of mitochondrial FAD transport and FAD synthesis are associated with myopathic phenotypes. Further research will be needed to unravel this enigma. Because of the potential beneficial effect of riboflavin in both primary and secondary flavocoenzyme deficiencies, early recognition of these disorders is imperative. Suprapharmacological doses of riboflavin are required for inborn errors associated with primary flavocoenzyme deficiency, and response to treatment may be more variable in patients with secondary deficiency.

\section{Compliance with ethics guidelines}

\section{Conflict of interest}

SB declares she has no conflict of interest. JC is a communicating editor of the Journal of Inherited Metabolic Disease. SR is an editor of the Journal of Inherited Metabolic Disease and JIMD Reports. 


\section{Acknowledgements and funding}

The Murdoch Children's Research Institute is supported by the Victorian Government's Operational Infrastructure Support Program. SR is supported by research grant funding from Great Ormond Street Hospital Children's Charity, the NIHR Great Ormond Street Hospital Biomedical Research Centre and the Lily Foundation.

\section{Ethics approval}

Ethics approval was not required for publication of this review.

\section{References:}

Auranen MA, Paetau A, Piirilä P et al (2017) Patient with multiple acyl-CoA dehydrogenation deficiency disease and FLAD1 mutations benefits from riboflavin therapy. Neuromuscular Disorders 27:581-584.

Barile M, Giancaspero TA, Leone P, Galluccio M, Indiveri C (2016) Riboflavin transport and metabolism in humans. J Inherit Metab Dis 39(4):545-57.

Bosch AM, Abeling NG, Ijlst L et al (2011) Brown-Vialetto-Van Laere and Fazio Londe syndrome is associated with a riboflavin transporter defect mimicking mild MADD: a new inborn error of metabolism with potential treatment. J Inherit Metab Dis 34: 159-164. 
Brizio C, Galluccio M, Wait R et al (2006) Over-expression in Escherichia coli and characterization of two recombinant isoforms of human FAD synthetase. Biochem Biophys Res Commun 344(3):1008-1016.

Buehler BA (2011) Vitamin B2: Riboflavin Journal of Evidence-Based Complementary \& Alternative Medicine 16(2): 88-90.

Carrozzo R, Torraco A, Fiermonte G et al (2014) Riboflavin responsive mitochondrial myopathy is a new phenotype of dihydrolipoamide dehydrogenase deficiency. The chaperonlike effect of vitamin B2. Mitochondrion 18: 49-57.

Chastain JL,McCormick DB (1987) Flavin catabolites: identification and quantitation in human urine. Am J Clin Nutr 46:830-834.

Collet M, Assouline Z, Bonnet et al (2016) High incidence and variable clinical outcome of cardiac hypertrophy due to ACAD9 mutations in childhood. European Journal of Human Genetics 24: 1112-1116.

De Meirleir LJ, Garcia-Cazorla A, Brivet M (2016) Disorders of Pyruvate Metabolism and the Tricarboxylic Acid Cycle. In Saudubray JM, Baumgartner MR, Walter J, $6^{\text {th }}$ eds. Inborn Metabolic Diseases Diagnosis and Treatment. Heidelberg: Springer Berlin, 194-195. 
Dewulf JP, Barrea C, Vincent MF (2016) Evidence of a wide spectrum of cardiac involvement due to ACAD9 mutations: Report on nine patients. Molecular Genetics and Metabolism 118:185-189.

Gempel K, Topaloglu H, Talim B, Schneiderat P, Schoser BG, Hans VH, Pálmafy B, Kale G, Tokatli A, Quinzii C, Hirano M, Naini A, DiMauro S, Prokisch H, Lochmüller H, Horvath R. The myopathic form of coenzyme Q10 deficiency is caused by mutations in the electron-transferring-flavoprotein dehydrogenase (ETFDH) gene. Brain. 2007 Aug;130(Pt 8):2037-44. Epub 2007 Apr 5. PubMed PMID: 17412732; PubMed Central PMCID: PMC4345103.

Ghezzi D, Sevrioukova I, Invernizzi F et al (2010) Severe X-linked mitochondrial encephalomyopathy associated with a mutation in apoptosis-inducing factor. Am J Hum Genet 86: 639-649.

Giancaspero TA, Busco G, Panebianco C et al (2013) FAD synthesis and degradation in the nucleus create a local flavin cofactor pool. J Biol Chem. 288(40):29069-29080.

Giancaspero TA, Colella M, Brizio C et al (2015) Remaining challenges in cellular flavin cofactor homeostasis and flavoprotein biogenesis. Front Chem. 22:3:30. 
Giancaspero TA, Dipalo E, Miccolis A, Boles E, Caselle M, Barile M (2014) Alteration of ROS homeostasis and decreased lifespan in S. cerevisiae elicited by deletion of the mitochondrial translocator FLX1. Biomed Res Int. 2014:101286.

Green P, Wiseman M, Crow YJ et al (2010) Brown-Vialetto-Van Laere syndrome, a pontobulbar palsy with deafness, is caused by mutations in C20ORF54. Am J Hum Genet 86: 485489.

Grünert SC (2014) Clinical and genetical heterogeneity of late-onset multiple acyl-coenzyme dehydrogenase deficiency. Orphanet Journal of Rare Diseases 9:117.

Heimer G, Eyal E, Zhu X et al (2018) Mutations in AIFM1 cause an X-linked childhood cerebellar ataxia partially responsive to riboflavin. Eur J Paediatr Neurol 22(1):93-101.

Hellebrekers DMEI, Sallevelt SCEH, Theunissen TEJ et al (2017) Novel SLC25A32 mutation in a patient with a severe neuromuscular phenotype. European Journal of Human Genetics 25: 886-888.

Henriques BJ, Rodrigues JV, Gomes CM (2013) Riboflavin and b-oxidation Flavoenzymes In Preedy VR. B Vitamins and Folate: Chemistry, Analysis, Function and Effects. Edition: Food and Nutritional Components in Focus No. 4. Cambridge: The Royal Society of Chemistry Publishing, chapter 37. 
Ho G, Yonezawa A, Masuda S et al (2011) Maternal riboflavin deficiency, resulting in transient neonatal-onset glutaric aciduria type 2 , is caused by a microdeletion in the riboflavin transporter gene GPR172B. Hum Mutat 32: E1976-E1984.

Hustad S, McKinley MC, McNulty H et al (2002) Riboflavin, flavin mononucleotide, and flavin adenine dinucleotide in human plasma and erythrocytes at baseline and after low-dose riboflavin supplementation. Clin Chem 48:1571-1577.

JoostenV, van Berkel WJ (2007) Flavoenzymes. Curr Opin Chem Biol 11:195-202.

Joza N, Pospisilik JA, Hangen E et al (2009) AIF: not just an apoptosis-inducing factor. Ann NY Acad Sci 1171: 2-11.

Karthikeyan S, Zhou Q, Mseeh F, Grishin NV, Osterman AL, Zhang H (2003) Crystal structure of human riboflavin kinase reveals a beta barrel fold and a novel active site arch. Structure 11: 265-273

Kennedy DO (2016) B Vitamins and the Brain: Mechanisms, Dose and Efficacy - A Review. Nutrients 8(2):68. 
Leone P, Galluccio M, Barbiroli A e al (2018) Bacterial Production, Characterization and Protein Modeling of a Novel Monofunctional Isoform of FAD Synthase in Humans: An Emergency Protein? Molecules 23:116-131.

Levy G, Jusko WJ (1966) Factors affecting the absorption of riboflavin in man. J Pharm Sci. 55(3):285-289.

Lienhart WD, Gudipati V, Macheroux P (2013) The human flavoproteome. Arch Biochem Biophys 535(2): 150-162.

Marashly ET, Bohlega SA (2017) Riboflavin has Neuroprotective Potential: Focus on Parkinson's Disease and Migraine. Front Neurol.8:333.

McCormick DB (1989) Two interconnected B vitamins: riboflavin and pyridoxine. Physiol Rev 69:1170-1198.

Mosegaard S, Bruun GH, Flyvbjerg KF et al (2017) intronic variation in SLC52A1 causes exon skipping and transient riboflavin-responsive multiple acyl-CoA dehydrogenation deficiency. Mol Genet Metab 122(4):182-188. 
Nouws J, Te Brinke H, Nijtmans LG, Houten SM (2014) ACAD9, a complex I assembly factor with a moonlighting function in fatty acid oxidation deficiencies. Hum Mol Genet 23: $1311-1319$.

O'Callaghan B, Bosch A, Houlden H (2019) An Update on the Genetics, Clinical Presentation and Pathomechanisms of Human Riboflavin Transporter Deficiency. J Inherit Metab Dis [in press]

Ogle RF, Christodoulou J, Fagan E et al (1997) Mitochondrial myopathy with tRNA ${ }^{\text {Leu(uuR) }}$ mutation and complex I deficiency responsive to riboflavin. J Pediatr 130:138-145.

Olsen RK, Andresen BS, Christensen E, Bross P, Skovby F, Gregersen N (2003) Clear relationship between ETF/ETFDH genotype and phenotype in patients with multiple acylCoA dehydrogenation deficiency. Hum Mutat 22: 12-23.

Olsen RK, Olpin SE, Andresen BS, Miedzybrodzka ZH, Pourfarzam M, Merinero B, Frerman FE, Beresford MW, Dean JC, Cornelius N, Andersen O, Oldfors A, Holme E, Gregersen N, Turnbull DM, Morris AA. ETFDH mutations as a major cause of riboflavin-responsive multiple acyl-CoA dehydrogenation deficiency. Brain. 2007 Aug;130(Pt 8):2045-54. Epub 2007 Jun 20. PubMed PMID: 17584774. 
Olsen RKJ, Konarikova E, Giancaspero TA et al (2016) Riboflavin-responsive and -nonresponsive mutations in FAD synthase cause multiple acyl-CoA dehydrogenase and combined respiratory-chain deficiency. Am J Hum Genet 98: 1130-1145

Pinto JT, Zempleni J (2016) Riboflavin. Adv Nutr 7(5):973-975.

Powers HJ (2003) Riboflavin (vitamin B-2) and health. Am J Clin Nutr 77(6):1352-6130.

Repp BM, Mastantuono E, Alston CL et al (2018) Clinical, biochemical and genetic spectrum of 70 patients with ACAD9 deficiency: is riboflavin supplementation effective? Orphanet J Rare Dis 13(1):120.

Rinaldi C, Grunseich C, Sevrioukova IF et al (2012) Cowchock syndrome is associated with a mutation in apoptosis-inducing factor. Am J Hum Genet 91(6):1095-1102.

Ryder B, Tolomeo M, Nochi Z, et al (2018) A Novel Truncating FLAD1 Variant, Causing Multiple Acyl-CoA Dehydrogenase Deficiency (MADD) in an 8-Year-Old Boy. JIMD Rep. 2018 Oct 12. doi:10.1007/8904_2018_139. [Epub ahead of print] PubMed PMID: 30311138.

Schiff M, Veauville-Merllie A, Acquaviva-Bourdain C (2016) SLC25A32 mutations and riboflavin-responsive exercise intolerance. New Eng J Med 374: 795-797.

This article is protected by copyright. All rights reserved. 
Smedts HP, Rakhshandehroo M, Verkleij-Hagoort AC et al (2008) Maternal intake of fat, riboflavin and nicotinamide and the risk of having offspring with congenital heart defects. Eur J Nutr 47:357-365.

Smith LD, Garg U (2017) Disorders of vitamins and cofactors. In Garg U, Smith LD, eds. Biomarkers in Inborn Errors of Metabolism, first ed. Elsevier.,361-397.

Spaan AN, Ijlst L, van Roermund CW, Wijburg FA, Wanders RJ, Waterham HR (2005) Identification of the human mitochondrial FAD transporter and its potential role in multiple acyl-CoA dehydrogenase deficiency. Mol Genet Metab 86(4):441-447

Taylor RW, Pyle A, Griffin H et al (2014) Use of whole-exome sequencing to determine the genetic basis of multiple mitochondrial respiratory chain complex deficiencies. JAMA 312 : $68-77$.

Titus SA, Moran RG (2000) Retrovirally mediated complementation of the glyB phenotype: cloning of a human gene encoding the carrier for entry of folates into mitochondria. J Biol Chem 275: 36811-36817.

Torchetti EM, Bonomi F, Galluccio M et al (2011) Human FAD synthase (isoform 2): A component of the machinery that delivers FAD to apo-flavoproteins. FEBS J. 278: 44344449 . 
Torchetti EM, Brizio C, Colella M et al (2010) Mitochondrial localization of human FAD synthetase isoform 1. Mitochondrion 10(3):263-273.

Yazdanpanah B, Wiegmann K, Tchikov V et al (2009) Riboflavin kinase couples TNF receptor 1 to NADPH oxidase. Nature 460: 1159-1163.

Yildiz Y, Olsen RKJ, Sivri HS, Akcoren Z, Nygaard HH, Tokatli A (2018) Post-Mortem Detection of FLAD1 Mutations in 2 Turkish Siblings with Hypotonia in Early Infancy. Neuromuscular Disorders. Available online 31 May 2018. DOI: 10.1016/j.nmd.2018.05.009

Yonezawa A, Inui K (2013) Novel riboflavin transporter family RFVT/SLC52: identification, nomenclature, functional characterization and genetic diseases of RFVT/SLC52. Mol Aspects Med 34(2-3):693-701.

Zempleni J, Galloway JR, McCormick DB (1996) Pharmacokinetics of orally and intravenously administered riboflavin in healthy humans. Am J Clin Nutr 63:54-66. 
Table 1: Reported cases with FAD synthase deficiency

\begin{tabular}{|c|c|c|c|c|c|c|c|c|}
\hline Patient* & Sex & Ethnicity & $\begin{array}{l}\text { Age at } \\
\text { onset }\end{array}$ & $\begin{array}{l}\text { Age at } \\
\text { death }\end{array}$ & Phenotype & Mutations & $\begin{array}{l}\text { Enzyme } \\
\text { domain } \\
\text { affected }\end{array}$ & Reference \\
\hline $1(30)$ & $\mathrm{M}$ & Turkish & $4 m$ & $8 \mathrm{~m}$ & $\begin{array}{l}\text { Respiratory insufficiency, } \\
\text { lipid myopathy }\end{array}$ & $\begin{array}{l}\text { Homozygous frameshift deletion } \\
\text { c.401_404delTTCT;p.(Phe134Cysfs } * 8 \text { ) } \\
\text { (originally reported as } \\
\text { c.397_400delTTCT) }\end{array}$ & MPTb & $\begin{array}{l}\text { Taylor et al } \\
2014\end{array}$ \\
\hline $2(\mathrm{~S} 1 \mathrm{a})$ & $\mathrm{F}$ & Turkish & $32 \mathrm{hr}$ & $3 d$ & Cardiorespiratory collapse & $\begin{array}{l}\text { Homozygous in-frame deletion } \\
\text { c.1484_1486delCCT;p.(Ser495del) }\end{array}$ & FADS & $\begin{array}{l}\text { Olsen et al } \\
2016\end{array}$ \\
\hline $3(\mathrm{~S} 1 \mathrm{~b})$ & $\mathrm{M}$ & Turkish & $3 m$ & $\begin{array}{l}\text { Alive } \\
22 \mathrm{y}\end{array}$ & $\begin{array}{l}\text { Cardiomyopathy in first year } \\
\text { of life, recurrent SVT (ICD), } \\
\text { riboflavin responsive }\end{array}$ & $\begin{array}{l}\text { Homozygous in-frame deletion } \\
\text { c.1484_1486delCCT;p.(Ser495del) }\end{array}$ & FADS & $\begin{array}{l}\text { Olsen et al } \\
2016\end{array}$ \\
\hline $4(\mathrm{~S} 2)$ & $\mathrm{F}$ & Finnish & $20 y$ & $\begin{array}{l}\text { Alive } \\
44 y\end{array}$ & $\begin{array}{l}\text { Exercise intolerance, } \\
\text { progressive muscle weakness }\end{array}$ & $\begin{array}{l}\text { Compound heterozygous } \\
\text { c.568_569dupGC;p.(Val191Glnfs*10) } \\
\text { and c.1588C>T;p.(Arg530Cys) }\end{array}$ & $\begin{array}{l}\text { MPTb, } \\
\text { FADS }\end{array}$ & $\begin{array}{l}\text { Olsen et al } \\
2016 ; \\
\text { Auranen et } \\
\text { al } 2017\end{array}$ \\
\hline $5(\mathrm{~S} 3)$ & $\mathrm{F}$ & Italian & $44 y$ & $\begin{array}{l}\text { Alive } \\
56 y\end{array}$ & $\begin{array}{l}\text { Exercise intolerance, gait } \\
\text { difficulties, bilateral foot } \\
\text { drop, weakness of both arms }\end{array}$ & $\begin{array}{l}\text { Compound heterozygous } \\
\text { c.836delT;p.(Phe279Serfs*45) and } \\
\text { c.1588C }>\text { T;p.(Arg530Cys) }\end{array}$ & $\begin{array}{l}\text { MPTb, } \\
\text { FADS }\end{array}$ & $\begin{array}{l}\text { Olsen et al } \\
2016\end{array}$ \\
\hline $6(\mathrm{~S} 4 \mathrm{a})$ & $\mathrm{F}$ & Turkish & $4 m$ & $\begin{array}{l}\text { Alive } \\
8 \mathrm{y}\end{array}$ & $\begin{array}{l}\text { Increasing muscle hypotonia } \\
\text { from infancy }\end{array}$ & $\begin{array}{l}\text { Homozygous frameshift indel } \\
\text { c.526_537delinsCA;p.(Ala176Glnfs*8) }\end{array}$ & MPTb & $\begin{array}{l}\text { Olsen et al } \\
2016\end{array}$ \\
\hline 7 (S4b) & $\mathrm{F}$ & Turkish & $8 m$ & $16 y$ & $\begin{array}{l}\text { Severe muscle hypotonia and } \\
\text { weakness, scoliosis, tube fed, } \\
\text { died of pneumonia }\end{array}$ & $\begin{array}{l}\text { Homozygous frameshift indel } \\
\text { c.526_537delinsCA;p.(Ala176Glnfs*8) }\end{array}$ & MPTb & $\begin{array}{l}\text { Olsen et al } \\
2016\end{array}$ \\
\hline
\end{tabular}

This article is protected by copyright. All rights reserved. 


\begin{tabular}{|c|c|c|c|c|c|c|c|c|}
\hline 8 (S5) & $\mathrm{M}$ & Turkish & $\begin{array}{l}\text { First } \\
\text { months of } \\
\text { life }\end{array}$ & $7 \mathrm{~m}$ & $\begin{array}{l}\text { Poor sucking and hypotonia in } \\
\text { first few months of life, } \\
\text { respiratory infection at } 6 \\
\text { months, recurrent episodes of } \\
\text { sudden cardiac arrest } \\
\text { (pacemaker), died of } \\
\text { multiorgan failure }\end{array}$ & $\begin{array}{l}\text { Homozygous frameshift deletion } \\
\text { c.401_404delTTCT;p.(Phe134Cysfs } * 8 \text { ) }\end{array}$ & MPTb & $\begin{array}{l}\text { Olsen et al } \\
2016\end{array}$ \\
\hline 9 (S6) & $\mathrm{F}$ & Italian & birth & $9 \mathrm{~m}$ & $\begin{array}{l}\text { Hypotonia, poor suck, } \\
\text { frequent vomiting, severe } \\
\text { respiratory insufficiency died } \\
\text { of septic shock following } \\
\text { pneumonia }\end{array}$ & $\begin{array}{l}\text { Compound heterozygous } \\
\text { c.324delG;p.(Arg109Alafs*3) and } \\
\text { c.498delC+c.508T }>\text { C; }(\text { p. } \\
\text { Ser167Profs*20+p.Phe170Leu) }\end{array}$ & MPTb & $\begin{array}{l}\text { Olsen et al } \\
2016\end{array}$ \\
\hline 10 (S7) & $\mathrm{F}$ & Turkish & $2 \mathrm{~m}$ & $4 \mathrm{~m}$ & $\begin{array}{l}\text { Severe neonatal hypotonia } \\
\text { and muscle weakness, } \\
\text { swallowing difficulties, } \\
\text { nasogastric feeding, sudden } \\
\text { respiratory deterioration at } 4 \\
\text { months, requiring non- } \\
\text { invasive ventilation and } \\
\text { leading to death }\end{array}$ & $\begin{array}{l}\text { Homozygous frameshift deletion } \\
\text { c.401_404delTTCT;p.(Phe134Cysfs } * 8 \text { ) }\end{array}$ & MPTb & $\begin{array}{l}\text { Olsen et al } \\
2016\end{array}$ \\
\hline $11(\mathrm{P} 1)$ & $\mathrm{M}$ & Turkish & $2 \mathrm{~m}$ & $6 \mathrm{~m}$ & $\begin{array}{l}\text { Progressive weakness and } \\
\text { decreased movements, poor } \\
\text { suck, tube feeding, persistent } \\
\text { vomiting, respiratory } \\
\text { insufficiency requiring } \\
\text { invasive ventilation from 2.5- }\end{array}$ & $\begin{array}{l}\text { Homozygous frameshift deletion } \\
\text { c.401_404delTTCT;p.(Phe134Cysfs } * 8 \text { ) }\end{array}$ & MPTb & $\begin{array}{l}\text { Yildiz et al } \\
2018\end{array}$ \\
\hline
\end{tabular}




\begin{tabular}{|c|c|c|c|c|c|c|c|c|}
\hline & & & & & $\begin{array}{l}5 \mathrm{~m} \text { (extubated after riboflavin } \\
\text { treatment commenced), died } \\
\text { of aspiration pneumonia }\end{array}$ & & & \\
\hline $12(\mathrm{P} 2)$ & $\mathrm{F}$ & Turkish & $2 \mathrm{~m}$ & $5 \mathrm{~m}$ & $\begin{array}{l}\text { Weak, floppy, no head } \\
\text { control, distinct hoarse cry, } \\
\text { poor suck, swallowing } \\
\text { difficulties, tube feeding, } \\
\text { vomiting, decreased } \\
\text { movements. }\end{array}$ & $\begin{array}{l}\text { Homozygous frameshift deletion } \\
\text { c.401_404delTTCT;p.(Phe134Cysfs } * 8)\end{array}$ & MPTb & $\begin{array}{l}\text { Yildiz et al } \\
2018\end{array}$ \\
\hline 13 & $\mathrm{M}$ & Palestinian & $\begin{array}{l}\text { NBS } \\
\text { diagnosis } \\
\text { of } \\
\text { MADD, } \\
\text { picked up } \\
\text { through } \\
\text { newborn } \\
\text { screening. }\end{array}$ & $\begin{array}{l}\text { Alive } \\
8 \mathrm{y}\end{array}$ & $\begin{array}{l}\text { Myopathic facies and nasal } \\
\text { speech first noted at } 3 \text { years, } \\
\text { after cessation of oral } \\
\text { riboflavin. Severe } \\
\text { velopharyngeal insufficiency } \\
\text { with speech difficulty at } 6 \\
\text { years. Evolving myopathy } \\
\text { with fatigue on chewing and } \\
\text { worsening exercise } \\
\text { intolerance at } 8 \text { years. }\end{array}$ & $\begin{array}{l}\text { Homozygous nonsense variant } \\
\text { c. } 745 \mathrm{C}>\mathrm{T} ; \mathrm{p} .\left(\operatorname{Arg} 249^{*}\right)\end{array}$ & MPTb & $\begin{array}{l}\text { Ryder et al } \\
2018\end{array}$ \\
\hline
\end{tabular}

Key: * number in brackets refers to identification number in original publication; d days; FADS FAD synthase domain; hr hours; ICD implantable cardioverter-defibrillator; indel insertion/deletion; $\mathrm{m}$ months; MADD multiple acylCoA dehydrogenase deficiency; MPTb molybdopterin binding domain; NBS newborn screening; SVT supraventricular tachycardia; y years 
Table 2 Biochemical abnormalities reported in FADS deficiency

\begin{tabular}{|l|l|l|l|}
\hline Abnormality & Number & $\%$ of those tested & Reference(s) \\
\hline $\begin{array}{l}\text { Increased plasma } \\
\text { acylcarnitines }\end{array}$ & $10 / 10$ & $100 \%$ & $\begin{array}{l}\text { Olsen } \text { et al 2016; } \\
\text { Yildiz } \text { et al } 2018 ; \\
\text { Ryder } \text { et al } 2018\end{array}$ \\
\hline $\begin{array}{l}\text { Increased urinary } \\
\text { organic acids }\end{array}$ & $9 / 9$ & $100 \%$ & $\begin{array}{l}\text { Olsen } \text { et al 2016; } \\
\text { Yildiz } \text { et al } 2018 ; \\
\text { Ryder } \text { et al } 2018\end{array}$ \\
\hline $\begin{array}{l}\text { Muscle } \boldsymbol{R C E} \\
\text { activities: }\end{array}$ & & & \\
\hline $\begin{array}{l}\text { Multiple RCE } \\
\text { deficiencies }\end{array}$ & $7 / 8$ & $87.5 \%$ & $\begin{array}{l}\text { Taylor } \text { et al 2014; } \\
\text { Olsen } \text { et al 2016; } \\
\text { Yildiz } \text { et al 2018 }\end{array}$ \\
\hline Normal RCEs & $1 / 8$ & $12.5 \%$ & Olsen et al 2016 \\
\hline $\begin{array}{l}\text { Decreased FADS } \\
\text { activity in } \\
\text { fibroblasts }\end{array}$ & $4 / 4$ & $100 \%$ & $\begin{array}{l}\text { Olsen } \text { et al 2016; } \\
\text { Ryder } \text { et al 2018 }\end{array}$ \\
\hline
\end{tabular}

This article is protected by copyright. All rights reserved. 
Table 3: Disorders of the human flavoproteome

\begin{tabular}{|c|c|c|c|c|c|}
\hline $\begin{array}{l}\text { Gene } \\
\text { symbol }\end{array}$ & Protein & $\begin{array}{l}\text { E.C. number } \\
\text { (BRENDA) }\end{array}$ & Cofactor & OMIM \# & Phenotype \\
\hline$A C A D 9$ & $\begin{array}{l}\text { Acyl-CoA } \\
\text { dehydrogenase } \\
\text { family, member } 9\end{array}$ & 1.3 .8 .8 & FAD & 611126 & $\begin{array}{l}\text { Infantile onset hypertrophic } \\
\text { cardiomyopathy with lactic } \\
\text { acidosis, later onset } \\
\text { exercise intolerance and } \\
\text { muscle weakness }\end{array}$ \\
\hline$A C A D M$ & $\begin{array}{l}\text { Acyl-CoA } \\
\text { dehydrogenase, } \\
\text { medium-chain }\end{array}$ & 1.3 .8 .7 & FAD & 201450 & $\begin{array}{l}\text { Hypoketotic } \\
\text { hypoglycaemic } \\
\text { encephalopathy with } \\
\text { prolonged fasting }\end{array}$ \\
\hline$A C A D S$ & $\begin{array}{l}\text { Acyl-CoA } \\
\text { dehydrogenase, } \\
\text { short-chain }\end{array}$ & 1.3 .8 .1 & FAD & 201470 & $\begin{array}{l}\text { Variable features, many } \\
\text { cases identified by NBS } \\
\text { asymptomatic }\end{array}$ \\
\hline$A C A D V L$ & $\begin{array}{l}\text { Acyl-CoA } \\
\text { dehydrogenase, very } \\
\text { long-chain }\end{array}$ & 1.3 .8 .9 & FAD & 201475 & $\begin{array}{l}\text { Neonatal cardiomyopathy, } \\
\text { infantile/early childhood } \\
\text { hypoketotic } \\
\text { hypoglycaemia, adult-onset } \\
\text { myopathy }\end{array}$ \\
\hline$A C O X 1$ & $\begin{array}{l}\text { Acyl-CoA oxidase, } \\
\text { palmitoyl, } \\
\text { peroxisomal }\end{array}$ & 1.3 .3 .6 & FAD & 264470 & $\begin{array}{l}\text { Peroxisomal fatty acid } \\
\text { oxidation disorder } \\
\text { resembling Zellweger } \\
\text { syndrome or neonatal } \\
\text { adrenoleukodystrophy }\end{array}$ \\
\hline$A C O X 2$ & $\begin{array}{l}\text { Acyl-CoA oxidase, } \\
\text { branched-chain, } \\
\text { peroxisomal }\end{array}$ & $\mathrm{N} / \mathrm{A}$ & FAD & 617308 & $\begin{array}{l}\text { Bile acid synthesis defect } \\
\text { type } 6\end{array}$ \\
\hline$A G P S$ & $\begin{array}{l}\text { Alkyldihydroxy- } \\
\text { acetonephosphate } \\
\text { synthase }\end{array}$ & 2.5 .1 .26 & FAD & 600121 & $\begin{array}{l}\text { Rhizomelic } \\
\text { chondrodysplasia punctata } \\
\text { type } 3\end{array}$ \\
\hline AIFMI & $\begin{array}{l}\text { Apoptosis-inducing } \\
\text { factor }\end{array}$ & $\mathrm{N} / \mathrm{A}$ & FAD & $\begin{array}{l}300816 \\
310490 \\
300614\end{array}$ & $\begin{array}{l}\text { Combined OXPHOS } \\
\text { deficiency, Cowchock } \\
\text { syndrome, X-linked } \\
\text { deafness }\end{array}$ \\
\hline COQ6 & $\begin{array}{l}\text { Coenzyme } \mathrm{Q}_{10} \\
\text { monooxygenase } 6\end{array}$ & N/A & FAD? & 614650 & $\begin{array}{l}\text { Primary coenzyme } \mathrm{Q}_{10} \\
\text { deficiency - infantile onset } \\
\text { severe progressive } \\
\text { nephrotic syndrome } \\
\text { (FSGS) and sensorineural } \\
\text { deafness }\end{array}$ \\
\hline
\end{tabular}




\begin{tabular}{|c|c|c|c|c|c|}
\hline CYB5R3 & $\begin{array}{l}\text { Cytochrome b5 } \\
\text { reductase } 3\end{array}$ & 1.6 .2 .2 & FAD & 250800 & Methaemoglobinaemia \\
\hline$C Y B B$ & $\begin{array}{l}\text { Cytochrome b(- } \\
245), \text { beta subunit }\end{array}$ & $\mathrm{N} / \mathrm{A}$ & FAD & 306400 & $\begin{array}{l}\text { Chronic granulomatous } \\
\text { disease, X-linked }\end{array}$ \\
\hline $\mathrm{D} 2 \mathrm{HGDH}$ & $\begin{array}{l}\text { D-2- } \\
\text { Hydroxyglutarate } \\
\text { dehydrogenase }\end{array}$ & 1.1 .99 .39 & FAD & 600721 & $\begin{array}{l}\text { D-2-hydroxyglutaric } \\
\text { aciduria - developmental } \\
\text { delay, epilepsy, hypotonia, } \\
\text { and dysmorphic features }\end{array}$ \\
\hline DHCR & $\begin{array}{l}24- \\
\text { Dehydrocholesterol } \\
\text { reductase }\end{array}$ & 1.3 .1 .72 & FAD & 602398 & $\begin{array}{l}\text { Desmosterolosis - multiple } \\
\text { congenital anomalies }\end{array}$ \\
\hline $\mathrm{DHODH}$ & $\begin{array}{l}\text { Dihydroorotate } \\
\text { dehydrogenase }\end{array}$ & 1.3 .5 .2 & FMN & 263750 & $\begin{array}{l}\text { Miller syndrome - postaxial } \\
\text { acrofacial dysostosis }\end{array}$ \\
\hline$D L D$ & $\begin{array}{l}\text { Dihydrolipoyl } \\
\text { dehydrogenase }\end{array}$ & 1.8 .1 .4 & FAD & 246900 & $\begin{array}{l}\text { Combined deficiency of } \\
\text { BCKDHC, PDHC and } \\
\text { KGDHC }\end{array}$ \\
\hline$D U O X 2$ & Dual oxidase 2 & 1.6 .3 .1 & FAD & 607200 & $\begin{array}{l}\text { Thyroid } \\
\text { dyshormonogenesis } 6\end{array}$ \\
\hline ETFA & $\begin{array}{l}\text { Electron transfer } \\
\text { flavoprotein alpha } \\
\text { subunit }\end{array}$ & $\mathrm{N} / \mathrm{A}$ & FAD & 231680 & MADD \\
\hline$E T F B$ & $\begin{array}{l}\text { Electron transfer } \\
\text { flavoprotein beta } \\
\text { subunit }\end{array}$ & $\mathrm{N} / \mathrm{A}$ & FAD & 231680 & MADD \\
\hline ETFDH & $\begin{array}{l}\text { Electron transfer } \\
\text { flavoprotein } \\
\text { dehydrogenase }\end{array}$ & 1.5 .5 .1 & FAD & 231680 & MADD \\
\hline$F D X R$ & $\begin{array}{l}\text { Ferredoxin } \\
\text { reductase }\end{array}$ & 1.18 .1 .2 & FAD & 617717 & $\begin{array}{l}\text { Auditory neuropathy and } \\
\text { optic atrophy }\end{array}$ \\
\hline FLAD1 & FAD synthase & 2.7 .7 .2 & FMN & 255100 & Lipid storage myopathy \\
\hline FMO3 & $\begin{array}{l}\text { Flavin-containing } \\
\text { monooxygenase } 3\end{array}$ & 1.14 .13 .8 & FAD & 602079 & Trimethylaminuria \\
\hline FOXRED1 & $\begin{array}{l}\text { FAD-dependent } \\
\text { oxidoreductase } \\
\text { domain-containing } \\
\text { protein } 1\end{array}$ & $\mathrm{~N} / \mathrm{A}$ & FAD & $\begin{array}{l}256000 \\
252010\end{array}$ & $\begin{array}{l}\text { Leigh syndrome, } \\
\text { mitochondrial complex I } \\
\text { deficiency }\end{array}$ \\
\hline$G C D H$ & $\begin{array}{l}\text { Glutaryl-CoA } \\
\text { dehydrogenase }\end{array}$ & 1.3 .8 .6 & FAD & 231670 & $\begin{array}{l}\text { Glutaric aciduria type I - } \\
\text { macrocephaly, basal } \\
\text { ganglia lesions and } \\
\text { progressive movement }\end{array}$ \\
\hline
\end{tabular}




\begin{tabular}{|c|c|c|c|c|c|}
\hline & & & & & disorder \\
\hline GFER & $\begin{array}{l}\text { Growth factor, erv1- } \\
\text { like }\end{array}$ & 1.8 .3 .2 & FAD & 613076 & $\begin{array}{l}\text { Mitochondrial myopathy, } \\
\text { congenital cataract, hearing } \\
\text { loss and developmental } \\
\text { delay }\end{array}$ \\
\hline$I V D$ & $\begin{array}{l}\text { Isovaleryl-CoA } \\
\text { dehydrogenase }\end{array}$ & 1.3 .8 .4 & FAD & 243500 & $\begin{array}{l}\text { Isovaleric acidemia - } \\
\text { recurrent episodes of } \\
\text { ketoacidosis }\end{array}$ \\
\hline$I Y D$ & $\begin{array}{l}\text { Iodotyrosine } \\
\text { deiodinase }\end{array}$ & N/A & FMN & 274800 & $\begin{array}{l}\text { Thyroid } \\
\text { dyshormonogenesis } 4\end{array}$ \\
\hline KDM1A & $\begin{array}{l}\text { Lysine-specific } \\
\text { demethylase 1A }\end{array}$ & $1.14 .11 . \mathrm{B} 1$ & FAD & 616728 & $\begin{array}{l}\text { Cleft palate, psychomotor } \\
\text { retardation, and distinctive } \\
\text { facial features }\end{array}$ \\
\hline$L 2 H G D H$ & $\begin{array}{l}\text { L-2- } \\
\text { Hydroxyglutarate } \\
\text { dehydrogenase }\end{array}$ & 1.1 .99 .2 & FAD & 236792 & $\begin{array}{l}\text { L-2-hydroxyglutaric } \\
\text { aciduria - PMR with } \\
\text { subcortical } \\
\text { leukoencephalopathy, } \\
\text { cerebellar atrophy, and } \\
\text { signal changes in putamina } \\
\text { and dentate nuclei on MRI }\end{array}$ \\
\hline MTHFR & $\begin{array}{l}5,10 \text {-Methylene- } \\
\text { tetrahydrofolate } \\
\text { reductase }\end{array}$ & 1.5 .1 .20 & FAD & 236250 & $\begin{array}{l}\text { Homocystinuria due to } \\
\text { MTHFR deficiency }\end{array}$ \\
\hline MTRR & $\begin{array}{l}\text { Methionine } \\
\text { synthase reductase }\end{array}$ & 1.16 .1 .8 & FMN & 236270 & $\begin{array}{l}\text { Cobalamin E deficiency } \\
\text { (homocystinuria and } \\
\text { megaloblastic anaemia) }\end{array}$ \\
\hline NDUFVI & $\begin{array}{l}\text { NADH-ubiquinone } \\
\text { oxidoreductase } \\
\text { flavoprotein } 1\end{array}$ & 1.6 .5 .3 & FMN & 252010 & $\begin{array}{l}\text { Mitochondrial complex I } \\
\text { deficiency }\end{array}$ \\
\hline NDUFV2 & $\begin{array}{l}\text { NADH-ubiquinone } \\
\text { oxidoreductase } \\
\text { flavoprotein } 2\end{array}$ & 1.6 .5 .3 & FMN & 252010 & $\begin{array}{l}\text { Mitochondrial complex I } \\
\text { deficiency }\end{array}$ \\
\hline$P N P O$ & $\begin{array}{l}\text { Pyridoxal 5'- } \\
\text { phosphate oxidase }\end{array}$ & 1.4 .3 .5 & FMN & 610090 & $\begin{array}{l}\text { Pyridoxal phosphate } \\
\text { responsive epilepsy }\end{array}$ \\
\hline POR & $\begin{array}{l}\text { Cytochrome P450 } \\
\text { oxidoreductase }\end{array}$ & 1.6 .2 .4 & FMN & 201750 & $\begin{array}{l}\text { Antley-Bixler syndrome } \\
\text { with genital anomalies and } \\
\text { disordered steroidogenesis }\end{array}$ \\
\hline$P P O X$ & $\begin{array}{l}\text { Protoporphyrinogen } \\
\text { oxidase }\end{array}$ & 1.3 .3 .4 & FAD & 176200 & Porphyria variegata \\
\hline PRODH & $\begin{array}{l}\text { Proline } \\
\text { dehydrogenase 1 }\end{array}$ & 1.5 .99 .8 & FAD & 239500 & Hyperprolinaemia type I \\
\hline
\end{tabular}




\begin{tabular}{|c|c|c|c|c|c|}
\hline SDHA & $\begin{array}{l}\text { Succinate } \\
\text { dehydrogenase } \\
\text { flavoprotein subunit } \\
\text { A }\end{array}$ & $\mathrm{N} / \mathrm{A}$ & FAD & $\begin{array}{l}256000 \\
252011 \\
614165\end{array}$ & $\begin{array}{l}\text { Leigh syndrome, } \\
\text { mitochondrial complex II } \\
\text { deficiency, paragangliomas }\end{array}$ \\
\hline SLC25A32 & $\begin{array}{l}\text { Mitochondrial } \\
\text { flavin nucleotide } \\
\text { transporter }\end{array}$ & $\mathrm{N} / \mathrm{A}$ & FAD & 616839 & $\begin{array}{l}\text { Riboflavin responsive } \\
\text { exercise intolerance }\end{array}$ \\
\hline SLC52A1 & $\begin{array}{l}\text { Riboflavin } \\
\text { transporter } 1 \\
\text { (RFVT1) }\end{array}$ & N/A & FAD & 615026 & Riboflavin deficiency \\
\hline SLC52A2 & $\begin{array}{l}\text { Riboflavin } \\
\text { transporter } 2 \\
\text { (RFVT2) }\end{array}$ & $\mathrm{N} / \mathrm{A}$ & FAD & 614707 & BVVL syndrome \\
\hline$S L C 52 A 3$ & $\begin{array}{l}\text { Riboflavin } \\
\text { transporter } 3 \\
\text { (RFVT3) }\end{array}$ & $\mathrm{N} / \mathrm{A}$ & FAD & 211530 & BVVL syndrome \\
\hline$S U G C T$ & $\begin{array}{l}\text { Succinyl-CoA: } \\
\text { glutarate-CoA } \\
\text { transferase }\end{array}$ & $\mathrm{N} / \mathrm{A}$ & FAD & 231690 & Glutaric aciduria type III \\
\hline TXNRD2 & $\begin{array}{l}\text { Thioredoxin } \\
\text { disulphide reductase } \\
2\end{array}$ & 1.8.1.9 & FAD & 617825 & $\begin{array}{l}\text { Familial glucocorticoid } \\
\text { deficiency }\end{array}$ \\
\hline$X D H$ & $\begin{array}{l}\text { Xanthine } \\
\text { dehydrogenase }\end{array}$ & 1.17 .1 .4 & FAD & 278300 & $\begin{array}{l}\text { Xanthinuria type I, } \\
\text { xanthine stones }\end{array}$ \\
\hline
\end{tabular}

List of flavoproteins selected from Table 1 in Lienhart et al 2013

Key: BCKDHC branched-chain alpha-keto acid dehydrogenase complex; BRENDA Comprehensive enzyme information system (http://www.brenda-enzymes.org); BVVL Brown-Vialetto-Van Laere; FAD flavin adenine dinucleotide; FMN flavin mononucleotide; FSGS focal segmental glomerulosclerosis; KGDHC alpha-ketoglutarate dehydrogenase complex; MADD multiple acylCoA dehydrogenase deficiency (see main text for clinical details); NBS newborn screening; OMIM Online Mendelian Inheritance in Man (https://www.omim.org); PDHC pyruvate dehydrogenase complex; PMR psychomotor retardation 


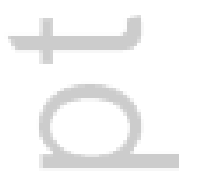

This article is protected by copyright. All rights reserved. 


\section{Figure legends}

\section{Figure 1a}

Dietary FAD and FMN are converted to riboflavin by hydrolases at the brush border of ileal enterocytes. Riboflavin is then absorbed into the intestine via apically expressed RFVT3 (also known as hRFT2) and released into portal blood by basolaterally expressed RFVT1 (also known as hRFT1) and RFVT2 (also known as hRFT3). Circulating plasma riboflavin is transported in blood bound to either albumin or immunoglobulins. RFVT2-mediated transport allows riboflavin uptake into the brain. The precise mechanism of import of riboflavin into the mitochondrial matrix has not been established to date. We have depicted RFVT? as a putative riboflavin transporter responsible for this step. Subsequent conversion into flavocoenzymes occurs in tissue cells, as shown in figure $1 \mathrm{~b}$. The mitochondrial FAD-transporter (FADT) is an inner mitochondrial membrane carrier that imports FAD from the cytosol into the mitochondria. The question mark indicates that FADT-mediated efflux of FAD from the mitochondrial matrix to the cytosol remains to be established. See also Barile et al, J Inher Metab Dis (2016): 39; 545-557.

\section{Figure 1b}

After cellular uptake of riboflavin, flavocoenzymes are formed by a sequential pathway involving riboflavin kinase and FAD synthase. Both steps require ATP. $\mathrm{Zn}^{2+}$ is the preferred metal ion which facilitates the ${ }^{3}$-phosphate exchange catalysed by riboflavin kinase, whilst $\mathrm{Mg}^{2+}$ is used in the adenylation catalysed by FAD synthase. 


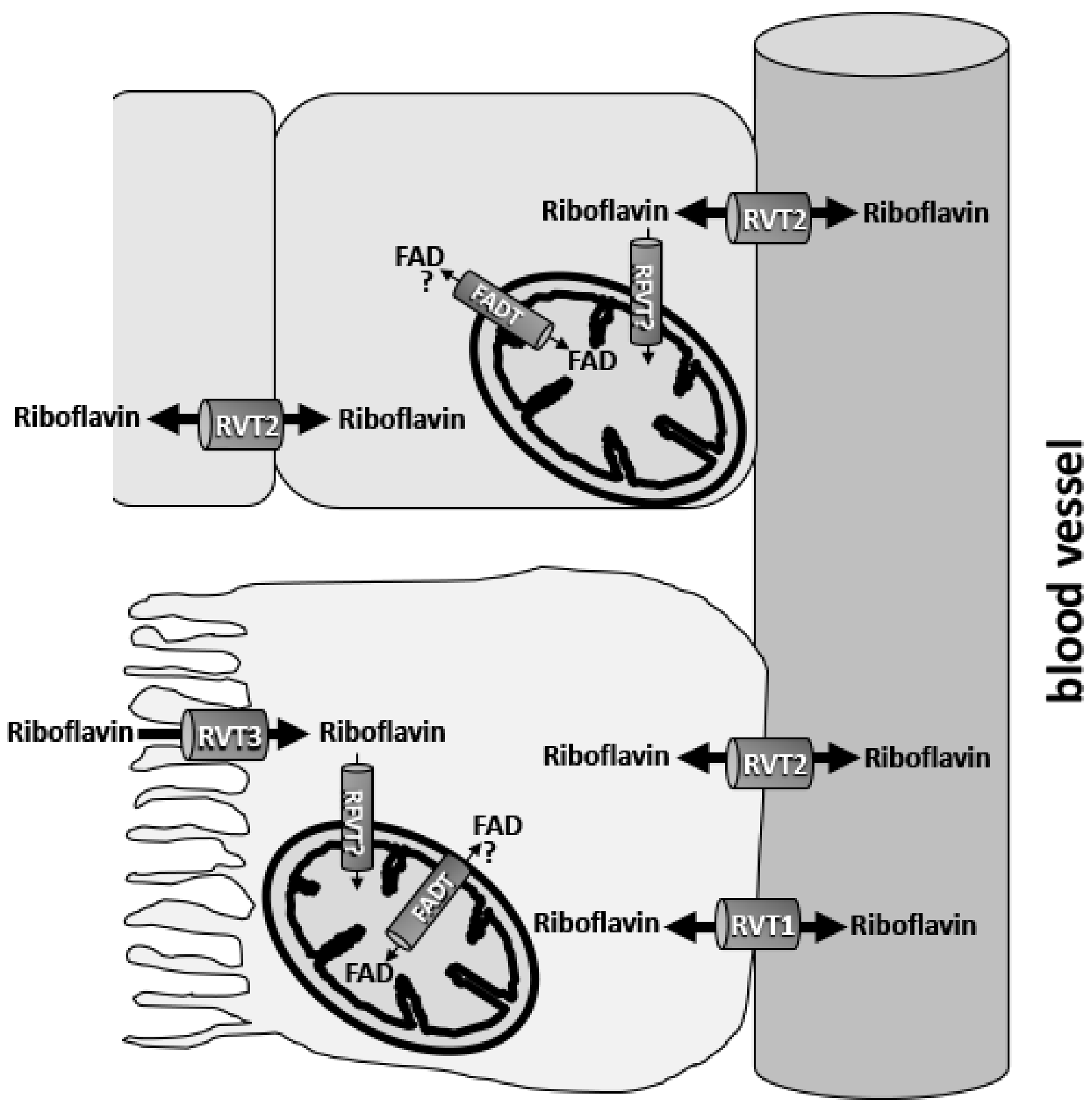

JIMD_12058_Balasubramaniam_fig 1a revised.tif 


\section{Disorders of Riboflavin Metabolism}

Shanti Balasubramaniam ${ }^{1,2,3,4}$ John Christodoulou $^{3,4,5,6}$, Shamima Rahman ${ }^{7,8}$

${ }^{1}$ Western Sydney Genetics Program, The Children's Hospital at Westmead, Sydney, NSW, Australia

${ }^{2}$ Kids Research Institute, The Children's Hospital at Westmead, Sydney, NSW, Australia ${ }^{3}$ Discipline of Genetic Medicine, Sydney Medical School, University of Sydney, Sydney, NSW, Australia

${ }^{4}$ Discipline of Child \& Adolescent Health, Sydney Medical School, University of Sydney, Sydney, NSW, Australia

${ }^{5}$ Neurodevelopmental Genomics Research Group, Murdoch Children's Research Institute, Melbourne, VIC, Australia

${ }^{6}$ Department of Paediatrics, Melbourne Medical School, University of Melbourne, Melbourne, VIC, Australia

${ }^{7}$ Mitochondrial Research Group, UCL Great Ormond Street Institute of Child Health, London, UK

${ }^{8}$ Metabolic Unit, Great Ormond Street Hospital NHS Foundation Trust, London, UK

\section{Corresponding author:}

Professor Shamima Rahman

Mitochondrial Research Group,

UCL Great Ormond Street Institute of Child Health,

London, United Kingdom

Phone: $+44(0) 2079052608$

Fax: $+44(0) 2074046191$

E-mail: shamima.rahman@ucl.ac.uk 


\begin{abstract}
Riboflavin (vitamin B2), a water-soluble vitamin, is an essential nutrient in higher organisms as it is not endogenously synthesized, with requirements being met principally by dietary intake. Tissue-specific transporter proteins direct riboflavin to the intracellular machinery responsible for the biosynthesis of the flavocoenzymes flavin mononucleotide (FMN) and flavin adenine dinucleotide (FAD). These flavocoenzymes play a vital role in ensuring the functionality of a multitude of flavoproteins involved in bioenergetics, redox homeostasis, DNA repair, chromatin remodelling, protein folding, apoptosis and other physiologically relevant processes. Hence, it is not surprising that the impairment of flavin homeostasis in humans may lead to multisystem dysfunction including neuromuscular disorders, anaemia, abnormal fetal development and cardiovascular disease. In this review we provide an overview of riboflavin absorption, transport, and metabolism. We then focus on the clinical and biochemical features associated with biallelic FLAD1 mutations leading to FAD synthase deficiency, the only known primary defect in flavocoenzyme synthesis, in addition to providing an overview of clinical disorders associated with nutritional deficiency of riboflavin and primary defects of riboflavin transport. Finally, we give a brief overview of disorders of the cellular flavoproteome. Because riboflavin therapy may be beneficial in a number of primary or secondary disorders of the cellular flavoproteome, early recognition and prompt management of these disorders is imperative.
\end{abstract}

\title{
Keywords:
}

Riboflavin metabolism, riboflavin transport, flavocoenzyme, flavoprotein, flavoproteome, FAD synthase deficiency, FLAD1 mutations, riboflavin responsive disorders. 


\section{Key messages:}

- Riboflavin is an essential nutrient in higher organisms since it is not endogenously synthesized and must be supplied through dietary intake.

- Primary defects of flavocoenzyme metabolism include genetically inherited disorders: a) involving enzymes in the synthetic pathway of essential cofactors FMN and FAD (i.e. FAD synthase deficiency; to date, no cases of human riboflavin kinase deficiency have been reported).

- b) disorders of riboflavin or flavocoenzyme transport.

- Secondary flavocoenzyme defects refer to the functional disruption of the cellular flavoproteome, i.e. cofactor dependent reactions which do not arise as a direct result of defects in the synthesis or transport of cofactors. Examples include multiple acylCoA dehydrogenase deficiency (MADD), glutaric aciduria type 1, and dihydrolipoamide dehydrogenase deficiency.

- Both primary and secondary flavocoenzyme deficiencies may present with abnormal biochemical features including characteristic elevations of plasma acylcarnitines and urinary organic acids.

- Riboflavin therapy may be beneficial in both primary and secondary flavocoenzyme deficiencies, hence early recognition of these disorders is imperative.

\section{Details of the Contributions of Individual Authors}

All three authors contributed to writing and critically revising the manuscript.

\section{Introduction}

Riboflavin (7,8-dimethyl-10-ribityl-isoalloxazine), also known as vitamin B2, plays a role in a wide range of metabolic pathways, because it is a precursor of the essential cofactors flavin 
mononucleotide (FMN) and flavin adenine dinucleotide (FAD) which are utilised in numerous enzymatic reactions. The human flavoprotein has a clear bias towards FADdependent enzymes which account for $84 \%$ of human flavoproteins, whilst only $16 \%$ use FMN as a cofactor (Joosten 2007, Lienhart et al 2013). Flavocoenzymes are able to catalyse one-electron and two-electron transfer reactions in oxidation-reduction processes, making them key players in a number of critical metabolic processes including the mitochondrial electron transport chain, mitochondrial and peroxisomal fatty acid $^{2}$-oxidation, the citric acid cycle, nitric oxide synthases, redox homeostasis and branched-chain amino acid catabolism. These flavoproteins are also important in chromatin remodelling, DNA repair, protein folding and apoptosis (Joosten et al 2007). Finally, flavoenzymes also play an important role in the biosynthesis or regulation of other essential cofactors and hormones, such as coenzyme A, coenzyme $\mathrm{Q}_{10}$, haem, steroids and thyroxine, as well as metabolism of other $\mathrm{B}$ vitamins (folate, pyridoxine/pyridoxal 5'-phosphate and niacin) (Lienhart et al 2013;Kennedy 2016).

\section{Dietary sources of riboflavin}

Riboflavin, as with other water-soluble B vitamins, must be obtained through the diet, since higher organisms have lost the ability to synthesize this molecule as opposed to various plants, fungi and bacteria. Endogenous synthesis of riboflavin (from ribulose-5-phosphate and GTP) by bacteria in the large intestine may contribute to some extent (Barile et al 2016). The recommended daily allowance (RDA) of riboflavin on average is $1.3 \mathrm{mg} /$ day for adult men and $1.1 \mathrm{mg} /$ day for women, with variations depending on age and reproductive status including pregnancy and lactation (Barile et al 2016). Milk and dairy products are the largest sources of riboflavin in the Western diet, making up 51\% of intake in preschool children, $35 \%$ in school children, $27 \%$ in adults, and 36\% in the elderly (Powers 2003). Due to the 
adequate availability of riboflavin in a wide variety of food sources, isolated deficiency is not prevalent in the general population, apart from rare genetic defects and malabsorptive conditions (Pinto and Zempleni 2016).

\section{Riboflavin transport and metabolism}

Riboflavin ingested in the diet exists either as free riboflavin or its protein bound form as flavoproteins containing FAD and FMN. Milk and eggs contain free riboflavin, but most other foods tend to predominantly contain FAD and FMN, which must be released from the carrier proteins to which they are bound. This is achieved by protein denaturation in the stomach, and subsequent hydrolysis to free riboflavin by alkaline phosphatases and FMN/FAD pyrophosphatases in the brush border of the ileal enterocyte, allowing absorption in the small intestine (Henriques et al 2013).

Three human plasma membrane riboflavin transporters have been characterised (Figure 1a): RFVT1 (previously hRFT1), RFVT2 (hRFT3) and RFVT3 (hRFT2), encoded by SLC52A1, SLC52A2 and SLC52A3 respectively (Yonezawa and Inui 2013). These three transporters have different subcellular locations and tissue specificities (Yonezawa and Inui 2013; Barile et al 2016). Free riboflavin is transported into enterocytes via carrier-mediated uptake by RFVT3 (Yonezawa and Inui 2013), a saturable transport process at the apical membrane which is reported to be linear up to approximately $30 \mathrm{mg}$ riboflavin per meal (Levy 1966; McCormick 1989; Powers 2003), and beyond which little additional absorption of riboflavin occurs (Zempleni et al 1996; Powers 2003).

After cellular uptake, riboflavin is quickly transformed into its catalytically active cofactors by the action of two enzymes: riboflavin kinase (RFK) (EC 2.7.1.26) and FAD synthase 
(FADS) (EC 2.7.7.2) which catalyse FMN and FAD production respectively (Figure 1b). Riboflavin may be released into the portal blood and to the liver in its free form or as FMN after being transported by RFTV1 and RFVT2 which are embedded within the basolateral membrane of enterocytes (Barile et al 2016). Circulating plasma riboflavin is bound to either albumin or immunoglobulins or is converted into its coenzyme forms in erythrocytes or leukocytes. Median plasma concentrations for riboflavin, FMN and FAD have been reported to be 10.5, 6.6 and $74 \mathrm{nmol} / \mathrm{L}$ (Hustad et al 2002; Barile 2016).

- RFK is a ubiquitous rate-limiting flavokinase that catalyses the first step in FAD biosynthesis by phosphorylating riboflavin to form FMN (Karthikeyan et al 2003), which is subsequently adenylated by FADS to generate FAD (Brizio et al 2006). The crystal structure of human RFK revealed a six-stranded antiparallel beta-barrel core that complexes with both riboflavin and ADP (Karthikeyan et al 2003). RFK has also been shown to be a tumour necrosis factor (TNF) receptor-1 (TNFR1)-binding protein that physically and functionally couples TNFR1 to NADPH oxidase. TNF, through the activation of RFK enhances the incorporation of FAD into NADPH oxidase, which is critical for the assembly and activation of NADPH oxidase (Yazdanpanah et al 2009).

FADS catalyses the adenylation of FMN to FAD (Brizio et al 2006). In humans, distinct isoforms of this enzyme are distributed in different subcellular compartments and generated by alternative splicing of the FLAD1 gene (Torchetti et al 2010; Giancaspero et al 2013; Barile et al 2016; Olsen et al 2016). The most abundant of these isoforms, i.e. the cytosolic enzyme or isoform 2, has been shown to be a bifunctional enzyme with both FAD synthase and hydrolase activity (Giancaspero et al 2015). The enzyme contains an N-terminal molybdopterin binding (MPTb) domain which has FAD hydrolase activity, and a C-terminal 
3'-phosphoadenosine-5' -phosphosulphate (PAPS) reductase domain, which is sufficient to catalyse FAD synthesis and so has been renamed the FADS domain (Giancaspero et al 2015; Olsen et al 2016). Very recently, a novel FADS6 isoform containing only the PAPS reductase domain was discovered in patients harbouring frameshift mutations of FLADl (Leone et al 2018). Functional characterisation suggested that FADS6 may represent an "emergency" enzyme that provides cytosolic FAD synthesizing capacity in the presence of biallelic FLADI frameshift variants, thus allowing survival of affected patients. The FAD synthesizing activity of FADS6 is approximately 70\% that of human FADS2 (Torchetti et al 2011); however, its catalytic efficiency $(\mathrm{kcat} / \mathrm{Km})$ is higher due to a lower FADS6 Km for both FMN and ATP compared to FADS2. FADS6 is expected to be only cytosolic since it lacks the mitochondrial targeting peptide, and might regulate the mitochondrial FAD transporter direction of transport by ensuring FAD import to facilitate flavoprotein biogenesis (Leone et al 2018).

SLC25A32 is an inner mitochondrial membrane carrier that imports FAD from the cytosol into mitochondria (mitochondrial FAD- transporter; Figure 1b), where it is needed for the many FAD-dependent mitochondrial enzymes, including components of the respiratory chain and the fatty acid beta oxidation spiral. SLC25A32 was initially identified as a mitochondrial folate transporter (Titus and Moran 2000), before it was demonstrated to be the human orthologue of the yeast mitochondrial FAD transporter FLX1 (Spaan et al 2005). The mitochondrial translocator FLX1 catalyzes the bidirectional movement of the redox cofactor FAD across the mitochondrial membrane (Giancaspero et al 2014). The possibility that SLC25A32 regulates FAD efflux from the mitochondrial matrix to the cytosol has been neither confirmed nor excluded in human cells. 
Unbound flavins are rapidly hydrolysed to free riboflavin and excreted in urine. Riboflavin is not stored in the body, hence any intake in excess of tissue requirements or which surpasses renal reabsorption, is eliminated in the urine as riboflavin or its catabolites 7-alpha-hydroxy riboflavin, 10-hydroxyethylflavin and lumiflavin (Chastain and McCormick 1987; Barile 2016). This means that riboflavin has a relatively low toxicity even at supra-pharmacological doses (Barile et al 2016).

\section{Riboflavin disorders in humans}

\subsection{Nutritional riboflavin deficiency}

Since riboflavin is not endogenously synthesized or stored in human tissues, adequate dietary intake is imperative. Clinical problems associated with dietary deficiency of riboflavin, also known as ariboflavinosis, include night blindness, cataracts, fatigue, anaemia, growth retardation, migraines, peripheral neuropathy and dermatological symptoms (cheilosis, glossitis, scaly skin rashes confined to the scrotum and vulva, and chapped lips) (Smith and Garg 2017). Low riboflavin intake during pregnancy may be associated with an increased risk of congenital heart disease (Smedts et al 2008). Symptomatic riboflavin deficiency often occurs in association with deficiencies of other vitamins and has usually been attributed to dietary restriction, however it is potentially more widespread than currently recognised with an estimated $10-15 \%$ of the global population having an inherited restriction in their ability to absorb or utilise riboflavin (Kennedy 2016; Marashly et al 2017). Supplementation for prevention of ariboflavinosis is generally not necessary as adequate amounts of riboflavin are available in a healthy diet, except when the diet is very limited or other health issues are present. Riboflavin deficiency can however occur during lactation, phototherapy in infants, or as a consequence of coeliac disease, alcoholism, malignancies, or use of prescription drugs 
including phenothiazine-derived antipsychotic medications, the antimalarial drug quinacrine, phenobarbitol and the cancer chemotherapy agent adriamycin (Buehler 2011).

\subsection{Disorders of riboflavin transport}

\subsubsection{Brown-Vialetto-Van Laere syndrome}

Deficiency of the plasma membrane riboflavin transporters SLC52A2 (OMIM \#614707) and SLC52A3 (OMIM \#211530) are associated with genetic neuronopathies. These include type 1 riboflavin transporter deficiency neuronopathy caused by mutations in the SLC52A3 gene, also known as Brown-Vialetto-Van Laere syndrome-1 (BVVLS1), a progressive neurologic disorder with bulbar palsy and sensorineural deafness (Green et al 2010), or Fazio-Londe disease, a disorder similar to BVVLS1 but without sensorineural deafness (Bosch et al 2011). Type 2 riboflavin transporter deficiency neuronopathy, or Brown-Vialetto-Van Laere syndrome-2 (BVVLS2) is caused by mutations in SLC52A2. Haploinsufficiency of the SLC52A1 riboflavin transporter (OMIM \#615026) has been reported in two neonates, associated with maternal riboflavin deficiency, who developed transient severe symptoms resembling multiple acyl-CoA dehydrogenase deficiency (MADD), which resolved with oral supplementation of riboflavin (Ho et al 2011, Mosegaard et al 2017). Disorders of riboflavin transport are discussed in detail in the accompanying review in this issue of the Journal of Inherited Metabolic Disease (O’Callaghan et al 2019).

\subsubsection{Mitochondrial FAD transporter deficiency (OMIM \# 616839)}

Two patients with biallelic mutations in SLC25A32 encoding the mitochondrial FAD transporter have been reported (Schiff et al 2016, Hellebrekers et al 2017). The first was a 14year-old girl with riboflavin-responsive recurrent exercise intolerance and biochemical

This article is protected by copyright. All rights reserved. 
features of MADD (Schiff et al 2016). The second patient, 51 years old at the time of reporting, presented at 3 years with muscle weakness following an episode of influenza, and subsequently had progressive exercise intolerance in childhood, together with neurological symptoms including early-onset ataxia, myoclonus, dysarthria and dysphagia (Hellebrekers et al 2017). Muscle biopsy revealed ragged-red fibres, lipid storage and fibres with decreased staining for succinate dehydrogenase (SDH, FAD-dependent mitochondrial respiratory chain complex II) and cytochrome $c$ oxidase (COX, mitochondrial respiratory chain complex IV) in both patients. Deficiency of complex II was demonstrated in muscle from the second patient and cultured skin fibroblasts from the first patient (Hellebrekers et al 2017, Schiff et al 2016). Both patients had dramatic improvements in the clinical and biochemical abnormalities following oral riboflavin supplementation, including improved exercise tolerance and endurance (Schiff et al 2016, Hellebrekers et al 2017).

It is interesting that these patients have a MADD-like predominantly myopathic presentation, quite distinct from the neuropathic presentation of patients with mutations in the plasma membrane riboflavin transporters RFVT2 and RFVT3.

\subsection{Primary defect of riboflavin coenzyme metabolism}

\subsubsection{FAD synthase deficiency (OMIM \# 255100)}

In the last few years biallelic variants in FLADI encoding FADS have been identified as causing a potentially treatable neuromuscular disease manifesting with lipid storage myopathy and metabolic abnormalities suggestive of MADD, in association with multiple respiratory chain enzyme deficiencies (Taylor et al 2014, Olsen et al 2016). Thirteen patients have been reported in the literature to date, all but one patient (Ryder et al 2018) were identified by whole exome sequencing or through candidate gene panels, and their clinical features are summarised in Table 1 (Taylor et al 2014, Olsen et al 2016, Auranen et al 2017, 
Yildiz et al 2018). Onset was in infancy in eleven cases (85\%). Presenting features in almost all of these infants included hypotonia and severe muscle weakness leading to feeding difficulties (poor suck and swallow, frequently necessitating tube feeding) and respiratory insufficiency. One patient presented with cardiorespiratory collapse at 32 hours of age and died at 3 days (Olsen et al 2016). Another had cardiomyopathy in the first year of life and recurrent supraventricular tachycardias necessitating implantable cardioverter defibrillator placement. This infant had a dramatic response to riboflavin supplementation and was still alive at 22 years (Olsen et al 2016). Another infant who needed a pacemaker because of recurrent episodes of sudden cardiac arrest died of multiorgan failure at 7 months (Olsen et al 2016). Eight of the eleven cases with an infantile onset died, seven within the first 9 months of life and the eighth at 16 years (Taylor et al 2014, Olsen et al 2016, Yildiz et al 2018). Three children who presented in infancy were alive, two at 8 years and the third at 22 years, all treated with riboflavin. Two individuals presented in adult life, at 20 and 44 years, and were alive at 44 and 56 years respectively (Olsen et al 2016, Auranen et al 2017). The adultonset cases presented with exercise intolerance and progressive muscle weakness, with gait difficulties, bilateral foot drop and arm weakness in the older patient (Olsen et al 2016, Auranen et al 2017).

Blood and urine biochemistry in FADS deficiency are typical of riboflavin deficiency or MADD, with increased acylcarnitines and urinary organic acids in all cases tested (Table 2). Elevations of C4-C14 even chain acylcarnitines, C5 and C5-DC and C10:1, C14:1, C16:1, C18:1 and C18:2 have all been reported (Olsen et al 2016, Yildiz et al 2018). In one infant only C4 was elevated, and in another the acylcarnitine profile was normal on some occasions and abnormal on others (Olsen et al 2016). Increased urinary excretion of ethylmalonic, adipic, suberic, glutaric and methylsuccinic acids, hexanoylglycine and tiglylglycine has been 
observed (Olsen et al 2016, Yildiz et al 2018, Ryder et al 2018). There appears to be a characteristic muscle pathology in FADS deficiency, with pronounced lipid storage and a global decrease of COX and/or SDH histochemical staining (Taylor et al 2014). Ragged-red fibres have not been reported so far. Multiple respiratory chain enzyme deficiencies were observed in skeletal muscle biopsies in 7 of 8 cases tested, variably affecting complexes I, II, III and/or IV (Taylor et al 2014; Olsen et al 2016; Yildiz et al 2018). One case was said to have normal muscle respiratory chain enzyme activities (Olsen et al 2016). Decreased FADS activity was demonstrated in cultured skin fibroblasts from all four patients tested (Olsen et al 2016, Ryder et al 2018).

Nine of the 13 patients reported with FADS deficiency were Turkish, and three different homozygous FLAD1 mutations were observed in this population (Table 1). One mutation appears to be particularly prevalent, suggesting a possible founder effect. This is a $4 \mathrm{bp}$ deletion (c.401_404delTTCT, initially reported as c.397_400delTTCT) in the MPTb domain resulting in a frameshift and premature termination (p.(Phe134CysfsTer8)), and was observed in five infants from four families (Taylor et al 2014, Olsen et al 2016, Yildiz et al 2018). All five died between 4 and 8 months of age. A possible genotype-phenotype correlation has been suggested (Olsen et al 2016). Three of four individuals with at least one FADS domain mutation affecting a single amino acid had prolonged survival (alive at 22-56 years), although the fourth died at only 3 days of life. Conversely, all 8 patients with biallelic frameshift mutations in the MPTb domain died, almost all in infancy (Table 1). One individual diagnosed with MADD on newborn screening was treated with riboflavin for the first 29 months of life, but developed a progressive myopathy from 3 years of age and was found to have a homozygous nonsense mutation in the MPTb domain of FLAD1 at 8 years (Ryder et al 2018). The nonsense mutation led to a truncated protein with some residual FADS activity 
(Ryder et al 2018). Clearly only a small number of patients with FLAD1 mutations have been identified so far, and so it is difficult to make firm genotype-phenotype predictions at present.

Riboflavin supplementation resulted in clinical improvements in seven of eight patients treated (Olsen et al 2016, Yildiz et al 2018, Ryder et al 2018), including dramatic amelioration of muscle symptoms and an increase in muscle strength, and improvements of the biochemical abnormalities in one adult patient who was further described in a subsequent report. This individual also received dietary therapy with high carbohydrate, moderate protein and low fat (max $20 \mathrm{~g}$ fat/day), which was reported to ameliorate her symptoms (Auranen et al 2017). Treatment with riboflavin at age three months in two patients homozygous for the c.401_404delTTCT mutation in FLAD1 seemed to result in mild improvements in spontaneous activity, muscle tone, vomiting and alertness, but failed to prevent disease progression; both infants died before the age of 6 months (Yildiz et al 2018). It has been proposed that biallelic frameshift mutations in exon 2 of FLAD1 may be partially responsive to riboflavin supplementation (Olsen et al 2016), but the doses used so far have not led to long-term survival (Yildiz et al 2018). Studies of the overexpressed mutant proteins in Escherichia coli demonstrated that FAD supplementation significantly improved protein stability and /or folding of the mutated proteins, supporting a chaperone-like action of FAD (Olsen et al 2016). Of note, riboflavin treatment initiated in the newborn period may have attenuated an otherwise severe phenotype in an 8-year-old boy with a homozygous nonsense mutation in FLADI who initially presented with MADD biochemical abnormalities in the newborn screening test (Ryder et al 2018).

In summary, all patients with FADS deficiency should receive high-dose riboflavin therapy, and if the diagnosis is suspected riboflavin supplementation should be implemented whilst 
awaiting mutational analysis, with subsequent withdrawal if the diagnosis is not confirmed. The benefits of early diagnosis and treatment are exemplified by discordant outcome between treated and untreated siblings (Olsen et al 2016). The potential avenue of prenatal or presymptomatic postnatal treatment with high dose riboflavin that may prevent or ameliorate the disease symptoms has been raised but requires further exploration. Other future therapeutic strategies include enhancing the activity of the truncated FADS isoform 6 (Leone et al 2018) or the mitochondrial FAD transporter in those patients who do not benefit from riboflavin (Yildiz et al 2018).

\subsubsection{Riboflavin kinase deficiency}

RFK deficiency has never been reported in humans. It is possible that this might be incompatible with life since, as discussed above, RFK is the rate-limiting step in the synthesis of FAD, an essential cofactor for many cellular reactions. In support of this hypothesis, RFK deficiency was embryonic lethal before day 7.5 of gestation in a knockout mouse model (Yazdanpanah et al 2009). However, it is possible that hypomorphic $R F K$ mutations may result in a clinical presentation similar to that seen in patients with FLADI mutations. Whole exome and whole genome sequencing of undiagnosed patients with lipid storage myopathies and MADD-like clinical presentations may identify affected cases in the fullness of time.

\subsection{Disorders of the human flavoproteome}

The human flavoproteome has been reported to comprise at least 90 distinct proteins, with diverse roles as indicated above (also see Table 3) (Lienhart et al 2013). Human diseases have been associated with approximately half of these proteins and are clinically extremely heterogeneous, including Leigh syndrome and other mitochondrial respiratory chain disorders, mitochondrial and peroxisomal fatty acid oxidation disorders, pyridoxal phosphate 
responsive epilepsy, trimethylaminuria, rhizomelic chondrodysplasia punctata, porphyria variegata, chronic granulomatous disease, and defects of steroidogenesis and bile acid synthesis (Table 3). Riboflavin responsiveness has not been reported for many of these disorders, and for several conditions it is not clear whether therapeutic trials of pharmacological doses of riboflavin have been explored as potential treatments. A full description of all disorders of the human flavoproteome is beyond the scope of this review, but some of the mitochondrial disorders associated with riboflavin responsiveness are discussed briefly below.

MADD (OMIM \#231680) is caused by deficiency of electron-transfer flavoprotein dehydrogenase (encoded by $E T F D H$ ) or of one of the two electron-transfer flavoproteins (encoded by $E T F A$ and $E T F B$ ) which transfer electrons from acyl-CoA dehydrogenases to coenzyme $\mathrm{Q}_{10}$ in the mitochondrial respiratory chain (Olsen et al 2003). Clinical phenotypes associated with MADD are heterogeneous and have been classified into a severe neonatal onset multsystemic form and a milder later onset myopathic form. The latter is the most frequent clinical presentation, manifesting as exercise intolerance, occasionally respiratory insufficiency or rhabdomyolysis and is typically riboflavin responsive (Olsen et al 2007; Grünert 2014). Other cases may have a secondary coenzyme $\mathrm{Q}_{10}$ deficiency (Gempel et al 2007). Riboflavin supplementation has successfully ameliorated clinical symptoms and metabolic abnormalities in over $95 \%$ of patients with late-onset MADD patients, the majority of whom (93\%) have ETFDH mutations (Grünert 2014).

ACAD9 is a bifunctional enzyme primarily involved in complex I biogenesis, with an additional moonlighting function in fatty acid oxidation (FAO) (Nouws et al 2014). The major clinical presentations of ACAD9 deficiency are early-onset cardiac involvement 
ranging from isolated electrical hypertrophy to severe hypertrophic, dilated or combined cardiomyopathy, exercise intolerance and myopathy, lactic acidosis and neurological abnormalities including stroke-like episodes, ataxic gait, bradykinesia and bradylalia (Dewulf et al 2016). $A C A D 9$ mutations are the most frequent cause of hypertrophic cardiomyopathy with isolated complex I deficiency (Collet et al 2016). Riboflavin has been shown to directly increase ACAD9 protein levels and rescue complex I assembly and may also function as a chemical chaperone by improving the folding of specific ACAD9 mutant proteins (Nouws et al 2014). Therapeutic response to riboflavin supplementation was reported in $65 \%$ of treated patients in one large multinational cohort of ACAD9 deficiency (Repp et al 2018). Riboflavin responsiveness has also been reported in other forms of complex I deficiency (Ogle et al 1997). It is possible that FMN may stabilise the complex I holoenzyme.

Dihydrolipoamide dehydrogenase (also known as E3, encoded by $D L D$ ) is a flavoprotein shared by three mitochondrial \pm -ketoacid dehydrogenase multi-enzyme complexes: pyruvate dehydrogenase complex (PDHc), \pm -ketoglutarate dehydrogenase complex (KGDC) and branched-chain \pm -keto acid dehydrogenase complex (BCKDC). The metabolic derangements reflect PDHC and TCA cycle defects, and include increased blood lactate and pyruvate, elevated plasma alanine, branched-chain amino acids and increased urinary lactic, pyruvic, 2ketoglutaric, and branched-chain 2-hydroxy- and 2-ketoacid (De Meirleir et al 2016). The phenotypic spectrum of DLD deficiency is variable, and encompasses early onset encephalopathy with progressive hypotonia, failure to thrive, hypoglycemia, ketoacidosis and encephalopathy, Leigh syndrome, and a recurrent Reye-like presentation with normal intellect and no residual neurologic deficits between acute metabolic episodes (Quinonez et el 2014). A riboflavin-responsive myopathic phenotype with exertional fatigue, intermittent elevation of blood lactate, ketoacidosis, elevation of creatine kinase and mitochondrial proliferation has also 
been described (Carrozzo et al 2014). Riboflavin supplementation led to complete resolution of muscle weakness, improvement of metabolic abnormalities, partial restoration of the DLD protein, and reduced ROS production in fibroblasts, supporting a chaperone-like effect of riboflavin promoting DLD protein stability and folding (Carrozzo et al 2014).

The X-linked AIFMI gene encodes apoptosis-inducing factor (AIF), a mitochondrial flavoprotein with a dual role as an FAD-dependent NADH oxidoreductase involved in oxidative phosphorylation and redox control and also as a caspase-independent death effector mediating programmed cell death when translocating from mitochondria to the nucleus on exposure to apoptotic stimuli (Joza et al 2009). AIFM1 mutations have been associated with a wide spectrum of clinical phenotypes ranging from a severe, early-onset mitochondrial encephalomyopathy with combined oxidative phosphorylation deficiency (OMIM \#300816) (Ghezzi et al 2010), to Cowchock syndrome, an X-linked Charcot-Marie-Tooth disease with axonal sensorimotor neuropathy, sensorineural deafness and cognitive impairment (CMTX4, OMIM \#310490) (Rinaldi et al 2012). Recently AIFM1 mutations have been reported to cause cerebellar ataxia partially responsive to riboflavin (Heimer et al 2018).

\section{Conclusions}

There is increasing evidence for a potential role of riboflavin in the prevention and treatment of various primary or secondary defects of maintenance of the cellular flavoproteome. In recent years, defects of FAD synthase in the flavocoenzyme biosynthetic pathway, the plasma membrane riboflavin transporters and the mitochondrial FAD transporter have all been linked to human disease. Human riboflavin kinase deficiency remains to be described. It is not known why the plasma membrane riboflavin transporter disorders manifest predominantly as a neuronopathy (BVVL) whereas defects of mitochondrial FAD transport and FAD synthesis 
are associated with myopathic phenotypes. Further research will be needed to unravel this enigma. Because of the potential beneficial effect of riboflavin in both primary and secondary flavocoenzyme deficiencies, early recognition of these disorders is imperative. Suprapharmacological doses of riboflavin are required for inborn errors associated with primary flavocoenzyme deficiency, and response to treatment may be more variable in patients with secondary deficiency.

\section{Compliance with ethics guidelines}

\section{Conflict of interest}

SB declares she has no conflict of interest. JC is a communicating editor of the Journal of Inherited Metabolic Disease. SR is an editor of the Journal of Inherited Metabolic Disease and JIMD Reports.

\section{Acknowledgements and funding}

The Murdoch Children's Research Institute is supported by the Victorian Government's Operational Infrastructure Support Program. SR is supported by research grant funding from Great Ormond Street Hospital Children's Charity, the NIHR Great Ormond Street Hospital Biomedical Research Centre and the Lily Foundation.

\section{Ethics approval}

Ethics approval was not required for publication of this review. 


\section{References:}

Auranen MA, Paetau A, Piirilä $P$ et al (2017) Patient with multiple acyl-CoA dehydrogenation deficiency disease and FLAD1 mutations benefits from riboflavin therapy. Neuromuscular Disorders 27:581-584.

Barile M, Giancaspero TA, Leone P, Galluccio M, Indiveri C (2016) Riboflavin transport and metabolism in humans. J Inherit Metab Dis 39(4):545-57.

Bosch AM, Abeling NG, Ijlst L et al (2011) Brown-Vialetto-Van Laere and Fazio Londe syndrome is associated with a riboflavin transporter defect mimicking mild MADD: a new inborn error of metabolism with potential treatment. J Inherit Metab Dis 34: 159-164.

Brizio C, Galluccio M, Wait R et al (2006) Over-expression in Escherichia coli and characterization of two recombinant isoforms of human FAD synthetase. Biochem Biophys Res Commun 344(3):1008-1016.

Buehler BA (2011) Vitamin B2: Riboflavin Journal of Evidence-Based Complementary \& Alternative Medicine 16(2): 88-90.

Carrozzo R, Torraco A, Fiermonte $G$ et al (2014) Riboflavin responsive mitochondrial myopathy is a new phenotype of dihydrolipoamide dehydrogenase deficiency. The chaperonlike effect of vitamin B2. Mitochondrion 18: 49-57.

Chastain JL,McCormick DB (1987) Flavin catabolites: identification and quantitation in human urine. Am J Clin Nutr 46:830-834. 
Collet M, Assouline Z, Bonnet et al (2016) High incidence and variable clinical outcome of cardiac hypertrophy due to ACAD9 mutations in childhood. European Journal of Human Genetics 24: 1112-1116.

De Meirleir LJ, Garcia-Cazorla A, Brivet M (2016) Disorders of Pyruvate Metabolism and the Tricarboxylic Acid Cycle. In Saudubray JM, Baumgartner MR, Walter J, $6^{\text {th }}$ eds. Inborn Metabolic Diseases Diagnosis and Treatment. Heidelberg: Springer Berlin, 194-195.

Dewulf JP, Barrea C, Vincent MF (2016) Evidence of a wide spectrum of cardiac involvement due to ACAD9 mutations: Report on nine patients. Molecular Genetics and Metabolism 118:185-189.

Gempel K, Topaloglu H, Talim B, Schneiderat P, Schoser BG, Hans VH, Pálmafy B, Kale G, Tokatli A, Quinzii C, Hirano M, Naini A, DiMauro S, Prokisch H, Lochmüller H, Horvath R. The myopathic form of coenzyme Q10 deficiency is caused by mutations in the electron-transferring-flavoprotein dehydrogenase (ETFDH) gene. Brain. 2007 Aug;130(Pt 8):2037-44. Epub 2007 Apr 5. PubMed PMID: 17412732; PubMed Central PMCID: PMC4345103.

Ghezzi D, Sevrioukova I, Invernizzi F et al (2010) Severe X-linked mitochondrial encephalomyopathy associated with a mutation in apoptosis-inducing factor. Am $\mathrm{J}$ Hum Genet 86: 639-649. 
Giancaspero TA, Busco G, Panebianco C et al (2013) FAD synthesis and degradation in the nucleus create a local flavin cofactor pool. J Biol Chem. 288(40):29069-29080.

Giancaspero TA, Colella M, Brizio C et al (2015) Remaining challenges in cellular flavin cofactor homeostasis and flavoprotein biogenesis. Front Chem. 22:3:30.

Giancaspero TA, Dipalo E, Miccolis A, Boles E, Caselle M, Barile M (2014) Alteration of ROS homeostasis and decreased lifespan in S. cerevisiae elicited by deletion of the mitochondrial translocator FLX1. Biomed Res Int. 2014:101286.

Green P, Wiseman M, Crow YJ et al (2010) Brown-Vialetto-Van Laere syndrome, a pontobulbar palsy with deafness, is caused by mutations in C20ORF54. Am J Hum Genet 86: 485489.

Grünert SC (2014) Clinical and genetical heterogeneity of late-onset multiple acyl-coenzyme dehydrogenase deficiency. Orphanet Journal of Rare Diseases 9:117.

Heimer G, Eyal E, Zhu X et al (2018) Mutations in AIFM1 cause an X-linked childhood cerebellar ataxia partially responsive to riboflavin. Eur J Paediatr Neurol 22(1):93-101.

Hellebrekers DMEI, Sallevelt SCEH, Theunissen TEJ et al (2017) Novel SLC25A32 mutation in a patient with a severe neuromuscular phenotype. European Journal of Human Genetics 25: 886-888. 
Henriques BJ, Rodrigues JV, Gomes CM (2013) Riboflavin and b-oxidation Flavoenzymes In Preedy VR. B Vitamins and Folate: Chemistry, Analysis, Function and Effects. Edition: Food and Nutritional Components in Focus No. 4. Cambridge: The Royal Society of Chemistry Publishing, chapter 37.

Ho G, Yonezawa A, Masuda S et al (2011) Maternal riboflavin deficiency, resulting in transient neonatal-onset glutaric aciduria type 2 , is caused by a microdeletion in the riboflavin transporter gene GPR172B. Hum Mutat 32: E1976-E1984.

Hustad S, McKinley MC, McNulty H et al (2002) Riboflavin, flavin mononucleotide, and flavin adenine dinucleotide in human plasma and erythrocytes at baseline and after low-dose riboflavin supplementation. Clin Chem 48:1571-1577.

JoostenV, van Berkel WJ (2007) Flavoenzymes. Curr Opin Chem Biol 11:195-202.

Joza N, Pospisilik JA, Hangen E et al (2009) AIF: not just an apoptosis-inducing factor. Ann NY Acad Sci 1171: 2-11.

Karthikeyan S, Zhou Q, Mseeh F, Grishin NV, Osterman AL, Zhang H (2003) Crystal structure of human riboflavin kinase reveals a beta barrel fold and a novel active site arch. Structure 11: 265-273

Kennedy DO (2016) B Vitamins and the Brain: Mechanisms, Dose and Efficacy - A Review. Nutrients 8(2):68. 
Leone P, Galluccio M, Barbiroli A e al (2018) Bacterial Production, Characterization and Protein Modeling of a Novel Monofunctional Isoform of FAD Synthase in Humans: An Emergency Protein? Molecules 23:116-131.

Levy G, Jusko WJ (1966) Factors affecting the absorption of riboflavin in man. J Pharm Sci. 55(3):285-289.

Lienhart WD, Gudipati V, Macheroux P (2013) The human flavoproteome. Arch Biochem Biophys 535(2): 150-162.

Marashly ET, Bohlega SA (2017) Riboflavin has Neuroprotective Potential: Focus on Parkinson's Disease and Migraine. Front Neurol.8:333.

McCormick DB (1989) Two interconnected B vitamins: riboflavin and pyridoxine. Physiol Rev 69:1170-1198.

Mosegaard S, Bruun GH, Flyvbjerg KF et al (2017) intronic variation in SLC52A1 causes exon skipping and transient riboflavin-responsive multiple acyl-CoA dehydrogenation deficiency. Mol Genet Metab 122(4):182-188.

Nouws J, Te Brinke H, Nijtmans LG, Houten SM (2014) ACAD9, a complex I assembly factor with a moonlighting function in fatty acid oxidation deficiencies. Hum Mol Genet 23: $1311-1319$. 
O'Callaghan B, Bosch A, Houlden H (2019) An Update on the Genetics, Clinical Presentation and Pathomechanisms of Human Riboflavin Transporter Deficiency. J Inherit Metab Dis [in press]

Ogle RF, Christodoulou J, Fagan E et al (1997) Mitochondrial myopathy with tRNA ${ }^{\text {Leu(uuR) }}$ mutation and complex I deficiency responsive to riboflavin. J Pediatr 130:138-145.

Olsen RK, Andresen BS, Christensen E, Bross P, Skovby F, Gregersen N (2003) Clear relationship between ETF/ETFDH genotype and phenotype in patients with multiple acylCoA dehydrogenation deficiency. Hum Mutat 22: 12-23.

Olsen RK, Olpin SE, Andresen BS, Miedzybrodzka ZH, Pourfarzam M, Merinero B, Frerman FE, Beresford MW, Dean JC, Cornelius N, Andersen O, Oldfors A, Holme E, Gregersen N, Turnbull DM, Morris AA. ETFDH mutations as a major cause of riboflavin-responsive multiple acyl-CoA dehydrogenation deficiency. Brain. 2007 Aug;130(Pt 8):2045-54. Epub 2007 Jun 20. PubMed PMID: 17584774.

Olsen RKJ, Konarikova E, Giancaspero TA et al (2016) Riboflavin-responsive and -nonresponsive mutations in FAD synthase cause multiple acyl-CoA dehydrogenase and combined respiratory-chain deficiency. Am J Hum Genet 98: 1130-1145

Pinto JT, Zempleni J (2016) Riboflavin. Adv Nutr 7(5):973-975.

Powers HJ (2003) Riboflavin (vitamin B-2) and health. Am J Clin Nutr 77(6):1352-6130. 
Repp BM, Mastantuono E, Alston CL et al (2018) Clinical, biochemical and genetic spectrum of 70 patients with ACAD9 deficiency: is riboflavin supplementation effective? Orphanet J Rare Dis 13(1):120.

Rinaldi C, Grunseich C, Sevrioukova IF et al (2012) Cowchock syndrome is associated with a mutation in apoptosis-inducing factor. Am J Hum Genet 91(6):1095-1102.

Ryder B, Tolomeo M, Nochi Z, et al (2018) A Novel Truncating FLAD1 Variant, Causing Multiple Acyl-CoA Dehydrogenase Deficiency (MADD) in an 8-Year-Old Boy. JIMD Rep. 2018 Oct 12. doi:10.1007/8904_2018_139. [Epub ahead of print] PubMed PMID: 30311138.

Schiff M, Veauville-Merllie A, Acquaviva-Bourdain C (2016) SLC25A32 mutations and riboflavin-responsive exercise intolerance. New Eng J Med 374: 795-797.

Smedts HP, Rakhshandehroo M, Verkleij-Hagoort AC et al (2008) Maternal intake of fat, riboflavin and nicotinamide and the risk of having offspring with congenital heart defects. Eur J Nutr 47:357-365.

Smith LD, Garg U (2017) Disorders of vitamins and cofactors. In Garg U, Smith LD, eds. Biomarkers in Inborn Errors of Metabolism, first ed. Elsevier.,361-397.

Spaan AN, Ijlst L, van Roermund CW, Wijburg FA, Wanders RJ, Waterham HR (2005) Identification of the human mitochondrial FAD transporter and its potential role in multiple acyl-CoA dehydrogenase deficiency. Mol Genet Metab 86(4):441-447 
Taylor RW, Pyle A, Griffin H et al (2014) Use of whole-exome sequencing to determine the genetic basis of multiple mitochondrial respiratory chain complex deficiencies. JAMA 312 : $68-77$.

Titus SA, Moran RG (2000) Retrovirally mediated complementation of the glyB phenotype: cloning of a human gene encoding the carrier for entry of folates into mitochondria. $\mathrm{J}$ Biol Chem 275: 36811-36817.

Torchetti EM, Bonomi F, Galluccio M et al (2011) Human FAD synthase (isoform 2): A component of the machinery that delivers FAD to apo-flavoproteins. FEBS J. 278: 44344449.

Torchetti EM, Brizio C, Colella M et al (2010) Mitochondrial localization of human FAD synthetase isoform 1. Mitochondrion 10(3):263-273.

Yazdanpanah B, Wiegmann K, Tchikov V et al (2009) Riboflavin kinase couples TNF receptor 1 to NADPH oxidase. Nature 460: 1159-1163.

Yildiz Y, Olsen RKJ, Sivri HS, Akcoren Z, Nygaard HH, Tokatli A (2018) Post-Mortem Detection of FLAD1 Mutations in 2 Turkish Siblings with Hypotonia in Early Infancy. Neuromuscular Disorders. Available online 31 May 2018. DOI: 10.1016/j.nmd.2018.05.009

Yonezawa A, Inui K (2013) Novel riboflavin transporter family RFVT/SLC52: identification, nomenclature, functional characterization and genetic diseases of RFVT/SLC52. Mol Aspects Med 34(2-3):693-701. 
Zempleni J, Galloway JR, McCormick DB (1996) Pharmacokinetics of orally and intravenously administered riboflavin in healthy humans. Am J Clin Nutr 63:54-66. 
Table 1: Reported cases with FAD synthase deficiency

\begin{tabular}{|c|c|c|c|c|c|c|c|c|}
\hline Patient* & Sex & Ethnicity & $\begin{array}{l}\text { Age at } \\
\text { onset }\end{array}$ & $\begin{array}{l}\text { Age at } \\
\text { death }\end{array}$ & Phenotype & Mutations & $\begin{array}{l}\text { Enzyme } \\
\text { domain } \\
\text { affected }\end{array}$ & Reference \\
\hline $1(30)$ & $\mathrm{M}$ & Turkish & $4 m$ & $8 m$ & $\begin{array}{l}\text { Respiratory insufficiency, } \\
\text { lipid myopathy }\end{array}$ & $\begin{array}{l}\text { Homozygous frameshift deletion } \\
\text { c.401_404delTTCT; p.(Phe134Cysfs } * 8 \text { ) } \\
\text { (originally reported as } \\
\text { c.397400delTTCT) }\end{array}$ & MPTb & $\begin{array}{l}\text { Taylor et al } \\
2014\end{array}$ \\
\hline $2(\mathrm{~S} 1 \mathrm{a})$ & $\mathrm{F}$ & Turkish & $32 \mathrm{hr}$ & $3 d$ & Cardiorespiratory collapse & $\begin{array}{l}\text { Homozygous in-frame deletion } \\
\text { c.1484_1486delCCT;p.(Ser495del) }\end{array}$ & FADS & $\begin{array}{l}\text { Olsen et al } \\
2016\end{array}$ \\
\hline 3 (S1b) & $\mathrm{M}$ & Turkish & $3 m$ & $\begin{array}{l}\text { Alive } \\
22 y\end{array}$ & $\begin{array}{l}\text { Cardiomyopathy in first year } \\
\text { of life, recurrent SVT (ICD), } \\
\text { riboflavin responsive }\end{array}$ & $\begin{array}{l}\text { Homozygous in-frame deletion } \\
\text { c.1484_1486delCCT;p.(Ser495del) }\end{array}$ & FADS & $\begin{array}{l}\text { Olsen et al } \\
2016\end{array}$ \\
\hline $4(\mathrm{~S} 2)$ & $\mathrm{F}$ & Finnish & $20 y$ & $\begin{array}{l}\text { Alive } \\
44 y\end{array}$ & $\begin{array}{l}\text { Exercise intolerance, } \\
\text { progressive muscle weakness }\end{array}$ & $\begin{array}{l}\text { Compound heterozygous } \\
\text { c.568_569dupGC;p.(Val191Glnfs*10) } \\
\text { and c.1588C>T;p.(Arg530Cys) }\end{array}$ & $\begin{array}{l}\text { MPTb, } \\
\text { FADS }\end{array}$ & $\begin{array}{l}\text { Olsen et al } \\
2016 ; \\
\text { Auranen et } \\
\text { al } 2017\end{array}$ \\
\hline $5(\mathrm{~S} 3)$ & $\mathrm{F}$ & Italian & $44 y$ & $\begin{array}{l}\text { Alive } \\
56 y\end{array}$ & $\begin{array}{l}\text { Exercise intolerance, gait } \\
\text { difficulties, bilateral foot } \\
\text { drop, weakness of both arms }\end{array}$ & $\begin{array}{l}\text { Compound heterozygous } \\
\text { c.836delT;p.(Phe279Serfs*45) and } \\
\text { c.1588C }>\text { T;p.(Arg530Cys) }\end{array}$ & $\begin{array}{l}\text { MPTb, } \\
\text { FADS }\end{array}$ & $\begin{array}{l}\text { Olsen et al } \\
2016\end{array}$ \\
\hline $6(\mathrm{~S} 4 \mathrm{a})$ & $\mathrm{F}$ & Turkish & $4 m$ & $\begin{array}{l}\text { Alive } \\
8 y\end{array}$ & $\begin{array}{l}\text { Increasing muscle hypotonia } \\
\text { from infancy }\end{array}$ & $\begin{array}{l}\text { Homozygous frameshift indel } \\
\text { c.526_537delinsCA;p.(Ala176Glnfs*8) }\end{array}$ & $\mathrm{MPTb}$ & $\begin{array}{l}\text { Olsen et al } \\
2016\end{array}$ \\
\hline 7 (S4b) & $\mathrm{F}$ & Turkish & $8 m$ & $16 y$ & $\begin{array}{l}\text { Severe muscle hypotonia and } \\
\text { weakness, scoliosis, tube fed, } \\
\text { died of pneumonia }\end{array}$ & $\begin{array}{l}\text { Homozygous frameshift indel } \\
\text { c.526_537delinsCA;p.(Ala176Glnfs*8) }\end{array}$ & MPTb & $\begin{array}{l}\text { Olsen et al } \\
2016\end{array}$ \\
\hline 8 (S5) & $\mathrm{M}$ & Turkish & $\begin{array}{l}\text { First } \\
\text { months of } \\
\text { life }\end{array}$ & $7 \mathrm{~m}$ & $\begin{array}{l}\text { Poor sucking and hypotonia in } \\
\text { first few months of life, } \\
\text { respiratory infection at } 6 \\
\text { months, recurrent episodes of } \\
\text { sudden cardiac arrest } \\
\text { (pacemaker), died of }\end{array}$ & $\begin{array}{l}\text { Homozygous frameshift deletion } \\
\text { c.401_404delTTCT;p.(Phe134Cysfs } * 8 \text { ) }\end{array}$ & MPTb & $\begin{array}{l}\text { Olsen et al } \\
2016\end{array}$ \\
\hline
\end{tabular}

This article is protected by copyright. All rights reserved. 


\begin{tabular}{|c|c|c|c|c|c|c|c|c|}
\hline & & & & & multiorgan failure & & & \\
\hline 9 (S6) & $\mathrm{F}$ & Italian & birth & $9 m$ & $\begin{array}{l}\text { Hypotonia, poor suck, } \\
\text { frequent vomiting, severe } \\
\text { respiratory insufficiency died } \\
\text { of septic shock following } \\
\text { pneumonia }\end{array}$ & $\begin{array}{l}\text { Compound heterozygous } \\
\text { c.324delG;p.(Arg109Alafs*3) and } \\
\text { c.498delC+c.508T }>\text { C; }(\text { p. } \\
\text { Ser167Profs*20+p.Phe170Leu) }\end{array}$ & MPTb & $\begin{array}{l}\text { Olsen et al } \\
2016\end{array}$ \\
\hline 10 (S7) & $F$ & Turkish & $2 \mathrm{~m}$ & $4 \mathrm{~m}$ & $\begin{array}{l}\text { Severe neonatal hypotonia } \\
\text { and muscle weakness, } \\
\text { swallowing difficulties, } \\
\text { nasogastric feeding, sudden } \\
\text { respiratory deterioration at } 4 \\
\text { months, requiring non- } \\
\text { invasive ventilation and } \\
\text { leading to death }\end{array}$ & $\begin{array}{l}\text { Homozygous frameshift deletion } \\
\text { c.401_404delTTCT;p.(Phe134Cysfs } * 8 \text { ) }\end{array}$ & MPTb & $\begin{array}{l}\text { Olsen et al } \\
2016\end{array}$ \\
\hline $11(\mathrm{P} 1)$ & $\mathrm{M}$ & Turkish & $2 \mathrm{~m}$ & $6 \mathrm{~m}$ & $\begin{array}{l}\text { Progressive weakness and } \\
\text { decreased movements, poor } \\
\text { suck, tube feeding, persistent } \\
\text { vomiting, respiratory } \\
\text { insufficiency requiring } \\
\text { invasive ventilation from } 2.5 \text { - } \\
5 \mathrm{~m} \text { (extubated after riboflavin } \\
\text { treatment commenced), died } \\
\text { of aspiration pneumonia }\end{array}$ & $\begin{array}{l}\text { Homozygous frameshift deletion } \\
\text { c.401_404delTTCT;p.(Phe134Cysfs } * 8 \text { ) }\end{array}$ & MPTb & $\begin{array}{l}\text { Yildiz et al } \\
2018\end{array}$ \\
\hline $12(\mathrm{P} 2)$ & $\mathrm{F}$ & Turkish & $2 m$ & $5 \mathrm{~m}$ & $\begin{array}{l}\text { Weak, floppy, no head } \\
\text { control, distinct hoarse cry, } \\
\text { poor suck, swallowing } \\
\text { difficulties, tube feeding, } \\
\text { vomiting, decreased } \\
\text { movements. }\end{array}$ & $\begin{array}{l}\text { Homozygous frameshift deletion } \\
\text { c.401_404delTTCT;p.(Phe134Cysfs } * 8 \text { ) }\end{array}$ & MPTb & $\begin{array}{l}\text { Yildiz et al } \\
2018\end{array}$ \\
\hline 13 & $\mathrm{M}$ & Palestinian & $\begin{array}{l}\text { NBS } \\
\text { diagnosis } \\
\text { of }\end{array}$ & $\begin{array}{l}\text { Alive } \\
8 \mathrm{y}\end{array}$ & $\begin{array}{l}\text { Myopathic facies and nasal } \\
\text { speech first noted at } 3 \text { years, } \\
\text { after cessation of oral }\end{array}$ & $\begin{array}{l}\text { Homozygous nonsense variant } \\
\text { c. } 745 \text { C }>\text { T;p. }(\text { Arg } 249 *)\end{array}$ & MPTb & $\begin{array}{l}\text { Ryder et al } \\
2018\end{array}$ \\
\hline
\end{tabular}

This article is protected by copyright. All rights reserved. 


\begin{tabular}{|l|l|l|l|l|}
\hline & $\begin{array}{l}\text { MADD, } \\
\text { picked up } \\
\text { through } \\
\text { newborn } \\
\text { screening. }\end{array}$ & $\begin{array}{l}\text { riboflavin. Severe } \\
\text { velopharyngeal insufficiency } \\
\text { with speech difficulty at } 6 \\
\text { years. Evolving myopathy } \\
\text { with fatigue on chewing and } \\
\text { worsening exercise } \\
\text { intolerance at } 8 \text { years. }\end{array}$ & \\
\hline
\end{tabular}

Key: * number in brackets refers to identification number in original publication; d days; FADS FAD synthase domain; hr hours; ICD implantable cardioverter-defibrillator; indel insertion/deletion; $\mathrm{m}$ months; MADD multiple acylCoA dehydrogenase deficiency; MPTb molybdopterin binding domain; NBS newborn screening; SVT supraventricular tachycardia; y years 
Table 2 Biochemical abnormalities reported in FADS deficiency

\begin{tabular}{|l|l|l|l|}
\hline Abnormality & Number & $\%$ of those tested & Reference(s) \\
\hline $\begin{array}{l}\text { Increased plasma } \\
\text { acylcarnitines }\end{array}$ & $10 / 10$ & $100 \%$ & $\begin{array}{l}\text { Olsen } \text { et al 2016; } \\
\text { Yildiz } \text { et al } 2018 ; \\
\text { Ryder } \text { et al } 2018\end{array}$ \\
\hline $\begin{array}{l}\text { Increased urinary } \\
\text { organic acids }\end{array}$ & $9 / 9$ & $100 \%$ & $\begin{array}{l}\text { Olsen } \text { et al 2016; } \\
\text { Yildiz } \text { et al } 2018 ; \\
\text { Ryder } \text { et al } 2018\end{array}$ \\
\hline $\begin{array}{l}\text { Muscle } \boldsymbol{R C E} \\
\text { activities: }\end{array}$ & & & \\
\hline $\begin{array}{l}\text { Multiple RCE } \\
\text { deficiencies }\end{array}$ & $7 / 8$ & $87.5 \%$ & $\begin{array}{l}\text { Taylor } \text { et al 2014; } \\
\text { Olsen } \text { et al 2016; } \\
\text { Yildiz } \text { et al 2018 }\end{array}$ \\
\hline Normal RCEs & $1 / 8$ & $12.5 \%$ & Olsen et al 2016 \\
\hline $\begin{array}{l}\text { Decreased FADS } \\
\text { activity in } \\
\text { fibroblasts }\end{array}$ & $4 / 4$ & $100 \%$ & $\begin{array}{l}\text { Olsen } \text { et al 2016; } \\
\text { Ryder } \text { et al 2018 }\end{array}$ \\
\hline
\end{tabular}

This article is protected by copyright. All rights reserved. 
Table 3: Disorders of the human flavoproteome

\begin{tabular}{|c|c|c|c|c|c|}
\hline $\begin{array}{l}\text { Gene } \\
\text { symbol }\end{array}$ & Protein & $\begin{array}{l}\text { E.C. number } \\
\text { (BRENDA) }\end{array}$ & Cofactor & OMIM \# & Phenotype \\
\hline$A C A D 9$ & $\begin{array}{l}\text { Acyl-CoA } \\
\text { dehydrogenase } \\
\text { family, member } 9\end{array}$ & 1.3 .8 .8 & FAD & 611126 & $\begin{array}{l}\text { Infantile onset hypertrophic } \\
\text { cardiomyopathy with lactic } \\
\text { acidosis, later onset } \\
\text { exercise intolerance and } \\
\text { muscle weakness }\end{array}$ \\
\hline$A C A D M$ & $\begin{array}{l}\text { Acyl-CoA } \\
\text { dehydrogenase, } \\
\text { medium-chain }\end{array}$ & 1.3 .8 .7 & FAD & 201450 & $\begin{array}{l}\text { Hypoketotic } \\
\text { hypoglycaemic } \\
\text { encephalopathy with } \\
\text { prolonged fasting }\end{array}$ \\
\hline$A C A D S$ & $\begin{array}{l}\text { Acyl-CoA } \\
\text { dehydrogenase, } \\
\text { short-chain }\end{array}$ & 1.3 .8 .1 & FAD & 201470 & $\begin{array}{l}\text { Variable features, many } \\
\text { cases identified by NBS } \\
\text { asymptomatic }\end{array}$ \\
\hline$A C A D V L$ & $\begin{array}{l}\text { Acyl-CoA } \\
\text { dehydrogenase, very } \\
\text { long-chain }\end{array}$ & 1.3 .8 .9 & FAD & 201475 & $\begin{array}{l}\text { Neonatal cardiomyopathy, } \\
\text { infantile/early childhood } \\
\text { hypoketotic } \\
\text { hypoglycaemia, adult-onset } \\
\text { myopathy }\end{array}$ \\
\hline$A C O X 1$ & $\begin{array}{l}\text { Acyl-CoA oxidase, } \\
\text { palmitoyl, } \\
\text { peroxisomal }\end{array}$ & 1.3 .3 .6 & FAD & 264470 & $\begin{array}{l}\text { Peroxisomal fatty acid } \\
\text { oxidation disorder } \\
\text { resembling Zellweger } \\
\text { syndrome or neonatal } \\
\text { adrenoleukodystrophy }\end{array}$ \\
\hline$A C O X 2$ & $\begin{array}{l}\text { Acyl-CoA oxidase, } \\
\text { branched-chain, } \\
\text { peroxisomal }\end{array}$ & N/A & FAD & 617308 & $\begin{array}{l}\text { Bile acid synthesis defect } \\
\text { type } 6\end{array}$ \\
\hline$A G P S$ & $\begin{array}{l}\text { Alkyldihydroxy- } \\
\text { acetonephosphate } \\
\text { synthase }\end{array}$ & 2.5 .1 .26 & FAD & 600121 & $\begin{array}{l}\text { Rhizomelic } \\
\text { chondrodysplasia punctata } \\
\text { type } 3\end{array}$ \\
\hline AIFMI & $\begin{array}{l}\text { Apoptosis-inducing } \\
\text { factor }\end{array}$ & $\mathrm{N} / \mathrm{A}$ & FAD & $\begin{array}{l}300816 \\
310490 \\
300614\end{array}$ & $\begin{array}{l}\text { Combined OXPHOS } \\
\text { deficiency, Cowchock } \\
\text { syndrome, X-linked } \\
\text { deafness }\end{array}$ \\
\hline COQ6 & $\begin{array}{l}\text { Coenzyme } \mathrm{Q}_{10} \\
\text { monooxygenase } 6\end{array}$ & $\mathrm{~N} / \mathrm{A}$ & FAD? & 614650 & $\begin{array}{l}\text { Primary coenzyme } \mathrm{Q}_{10} \\
\text { deficiency - infantile onset } \\
\text { severe progressive } \\
\text { nephrotic syndrome } \\
\text { (FSGS) and sensorineural } \\
\text { deafness }\end{array}$ \\
\hline CYB5R3 & $\begin{array}{l}\text { Cytochrome b5 } \\
\text { reductase } 3\end{array}$ & 1.6 .2 .2 & FAD & 250800 & Methaemoglobinaemia \\
\hline$C Y B B$ & $\begin{array}{l}\text { Cytochrome b(- } \\
245) \text {, beta subunit }\end{array}$ & N/A & FAD & 306400 & $\begin{array}{l}\text { Chronic granulomatous } \\
\text { disease, X-linked }\end{array}$ \\
\hline $\mathrm{D} 2 \mathrm{HGDH}$ & $\begin{array}{l}\text { D-2- } \\
\text { Hydroxyglutarate }\end{array}$ & 1.1 .99 .39 & FAD & 600721 & $\begin{array}{l}\text { D-2-hydroxyglutaric } \\
\text { aciduria - developmental }\end{array}$ \\
\hline
\end{tabular}




\begin{tabular}{|c|c|c|c|c|c|}
\hline & dehydrogenase & & & & $\begin{array}{l}\text { delay, epilepsy, hypotonia, } \\
\text { and dysmorphic features }\end{array}$ \\
\hline DHCR & $\begin{array}{l}24- \\
\text { Dehydrocholesterol } \\
\text { reductase }\end{array}$ & 1.3 .1 .72 & FAD & 602398 & $\begin{array}{l}\text { Desmosterolosis - multiple } \\
\text { congenital anomalies }\end{array}$ \\
\hline $\mathrm{DHODH}$ & $\begin{array}{l}\text { Dihydroorotate } \\
\text { dehydrogenase }\end{array}$ & 1.3 .5 .2 & FMN & 263750 & $\begin{array}{l}\text { Miller syndrome - postaxial } \\
\text { acrofacial dysostosis }\end{array}$ \\
\hline$D L D$ & $\begin{array}{l}\text { Dihydrolipoyl } \\
\text { dehydrogenase }\end{array}$ & 1.8 .1 .4 & FAD & 246900 & $\begin{array}{l}\text { Combined deficiency of } \\
\text { BCKDHC, PDHC and } \\
\text { KGDHC }\end{array}$ \\
\hline DUOX2 & Dual oxidase 2 & 1.6 .3 .1 & FAD & 607200 & $\begin{array}{l}\text { Thyroid } \\
\text { dyshormonogenesis } 6\end{array}$ \\
\hline ETFA & $\begin{array}{l}\text { Electron transfer } \\
\text { flavoprotein alpha } \\
\text { subunit }\end{array}$ & $\mathrm{N} / \mathrm{A}$ & FAD & 231680 & MADD \\
\hline$E T F B$ & $\begin{array}{l}\text { Electron transfer } \\
\text { flavoprotein beta } \\
\text { subunit }\end{array}$ & $\mathrm{N} / \mathrm{A}$ & FAD & 231680 & MADD \\
\hline ETFDH & $\begin{array}{l}\text { Electron transfer } \\
\text { flavoprotein } \\
\text { dehydrogenase }\end{array}$ & 1.5.5.1 & FAD & 231680 & MADD \\
\hline$F D X R$ & $\begin{array}{l}\text { Ferredoxin } \\
\text { reductase }\end{array}$ & 1.18 .1 .2 & FAD & 617717 & $\begin{array}{l}\text { Auditory neuropathy and } \\
\text { optic atrophy }\end{array}$ \\
\hline FLAD1 & FAD synthase & 2.7 .7 .2 & FMN & 255100 & Lipid storage myopathy \\
\hline$F M O 3$ & $\begin{array}{l}\text { Flavin-containing } \\
\text { monooxygenase } 3\end{array}$ & 1.14 .13 .8 & FAD & 602079 & Trimethylaminuria \\
\hline FOXREDI & $\begin{array}{l}\text { FAD-dependent } \\
\text { oxidoreductase } \\
\text { domain-containing } \\
\text { protein } 1\end{array}$ & $\mathrm{~N} / \mathrm{A}$ & FAD & $\begin{array}{l}256000 \\
252010\end{array}$ & $\begin{array}{l}\text { Leigh syndrome, } \\
\text { mitochondrial complex I } \\
\text { deficiency }\end{array}$ \\
\hline$G C D H$ & $\begin{array}{l}\text { Glutaryl-CoA } \\
\text { dehydrogenase }\end{array}$ & 1.3 .8 .6 & FAD & 231670 & $\begin{array}{l}\text { Glutaric aciduria type I- } \\
\text { macrocephaly, basal } \\
\text { ganglia lesions and } \\
\text { progressive movement } \\
\text { disorder }\end{array}$ \\
\hline GFER & $\begin{array}{l}\text { Growth factor, erv1- } \\
\text { like }\end{array}$ & 1.8 .3 .2 & FAD & 613076 & $\begin{array}{l}\text { Mitochondrial myopathy, } \\
\text { congenital cataract, hearing } \\
\text { loss and developmental } \\
\text { delay }\end{array}$ \\
\hline$I V D$ & $\begin{array}{l}\text { Isovaleryl-CoA } \\
\text { dehydrogenase }\end{array}$ & 1.3 .8 .4 & FAD & 243500 & $\begin{array}{l}\text { Isovaleric acidemia - } \\
\text { recurrent episodes of } \\
\text { ketoacidosis }\end{array}$ \\
\hline$I Y D$ & $\begin{array}{l}\text { Iodotyrosine } \\
\text { deiodinase }\end{array}$ & $\mathrm{N} / \mathrm{A}$ & FMN & 274800 & $\begin{array}{l}\text { Thyroid } \\
\text { dyshormonogenesis } 4\end{array}$ \\
\hline KDM1A & $\begin{array}{l}\text { Lysine-specific } \\
\text { demethylase 1A }\end{array}$ & 1.14.11.B1 & FAD & 616728 & $\begin{array}{l}\text { Cleft palate, psychomotor } \\
\text { retardation, and distinctive }\end{array}$ \\
\hline
\end{tabular}




\begin{tabular}{|c|c|c|c|c|c|}
\hline & & & & & facial features \\
\hline$L 2 H G D H$ & $\begin{array}{l}\text { L-2- } \\
\text { Hydroxyglutarate } \\
\text { dehydrogenase }\end{array}$ & 1.1 .99 .2 & FAD & 236792 & $\begin{array}{l}\text { L-2-hydroxyglutaric } \\
\text { aciduria-PMR with } \\
\text { subcortical } \\
\text { leukoencephalopathy, } \\
\text { cerebellar atrophy, and } \\
\text { signal changes in putamina } \\
\text { and dentate nuclei on MRI }\end{array}$ \\
\hline MTHFR & $\begin{array}{l}\text { 5,10-Methylene- } \\
\text { tetrahydrofolate } \\
\text { reductase }\end{array}$ & 1.5 .1 .20 & FAD & 236250 & $\begin{array}{l}\text { Homocystinuria due to } \\
\text { MTHFR deficiency }\end{array}$ \\
\hline MTRR & $\begin{array}{l}\text { Methionine } \\
\text { synthase reductase }\end{array}$ & 1.16 .1 .8 & FMN & 236270 & $\begin{array}{l}\text { Cobalamin E deficiency } \\
\text { (homocystinuria and } \\
\text { megaloblastic anaemia) }\end{array}$ \\
\hline NDUFV1 & $\begin{array}{l}\text { NADH-ubiquinone } \\
\text { oxidoreductase } \\
\text { flavoprotein } 1\end{array}$ & 1.6 .5 .3 & FMN & 252010 & $\begin{array}{l}\text { Mitochondrial complex I } \\
\text { deficiency }\end{array}$ \\
\hline NDUFV2 & $\begin{array}{l}\text { NADH-ubiquinone } \\
\text { oxidoreductase } \\
\text { flavoprotein } 2\end{array}$ & 1.6.5.3 & FMN & 252010 & $\begin{array}{l}\text { Mitochondrial complex I } \\
\text { deficiency }\end{array}$ \\
\hline$P N P O$ & $\begin{array}{l}\text { Pyridoxal 5'- } \\
\text { phosphate oxidase }\end{array}$ & 1.4 .3 .5 & FMN & 610090 & $\begin{array}{l}\text { Pyridoxal phosphate } \\
\text { responsive epilepsy }\end{array}$ \\
\hline$P O R$ & $\begin{array}{l}\text { Cytochrome P450 } \\
\text { oxidoreductase }\end{array}$ & 1.6 .2 .4 & FMN & 201750 & $\begin{array}{l}\text { Antley-Bixler syndrome } \\
\text { with genital anomalies and } \\
\text { disordered steroidogenesis }\end{array}$ \\
\hline$P P O X$ & $\begin{array}{l}\text { Protoporphyrinogen } \\
\text { oxidase }\end{array}$ & 1.3.3.4 & FAD & 176200 & Porphyria variegata \\
\hline PRODH & $\begin{array}{l}\text { Proline } \\
\text { dehydrogenase } 1\end{array}$ & 1.5 .99 .8 & FAD & 239500 & Hyperprolinaemia type I \\
\hline SDHA & $\begin{array}{l}\text { Succinate } \\
\text { dehydrogenase } \\
\text { flavoprotein subunit } \\
\text { A }\end{array}$ & N/A & FAD & $\begin{array}{l}256000 \\
252011 \\
614165\end{array}$ & $\begin{array}{l}\text { Leigh syndrome, } \\
\text { mitochondrial complex II } \\
\text { deficiency, paragangliomas }\end{array}$ \\
\hline$S L C 25 A 32$ & $\begin{array}{l}\text { Mitochondrial } \\
\text { flavin nucleotide } \\
\text { transporter }\end{array}$ & N/A & FAD & 616839 & $\begin{array}{l}\text { Riboflavin responsive } \\
\text { exercise intolerance }\end{array}$ \\
\hline$S L C 52 A 1$ & $\begin{array}{l}\text { Riboflavin } \\
\text { transporter } 1 \\
\text { (RFVT1) }\end{array}$ & N/A & FAD & 615026 & Riboflavin deficiency \\
\hline SLC52A2 & $\begin{array}{l}\text { Riboflavin } \\
\text { transporter } 2 \\
\text { (RFVT2) }\end{array}$ & N/A & FAD & 614707 & BVVL syndrome \\
\hline$S L C 52 A 3$ & $\begin{array}{l}\text { Riboflavin } \\
\text { transporter } 3 \\
\text { (RFVT3) }\end{array}$ & N/A & FAD & 211530 & BVVL syndrome \\
\hline SUGCT & $\begin{array}{l}\text { Succinyl-CoA: } \\
\text { glutarate-CoA }\end{array}$ & $\mathrm{N} / \mathrm{A}$ & FAD & 231690 & Glutaric aciduria type III \\
\hline
\end{tabular}




\begin{tabular}{|l|l|l|l|l|l|}
\hline & transferase & & & & \\
\hline TXNRD2 & $\begin{array}{l}\text { Thioredoxin } \\
\text { disulphide reductase } \\
2\end{array}$ & 1.8 .1 .9 & FAD & 617825 & $\begin{array}{l}\text { Familial glucocorticoid } \\
\text { deficiency }\end{array}$ \\
\hline XDH & $\begin{array}{l}\text { Xanthine } \\
\text { dehydrogenase }\end{array}$ & 1.17 .1 .4 & FAD & 278300 & $\begin{array}{l}\text { Xanthinuria type I, } \\
\text { xanthine stones }\end{array}$ \\
\hline
\end{tabular}

List of flavoproteins selected from Table 1 in Lienhart et al 2013

Key: BCKDHC branched-chain alpha-keto acid dehydrogenase complex; BRENDA Comprehensive enzyme information system (http://www.brenda-enzymes.org); BVVL Brown-Vialetto-Van Laere; FAD flavin adenine dinucleotide; FMN flavin mononucleotide; FSGS focal segmental glomerulosclerosis; KGDHC alpha-ketoglutarate dehydrogenase complex; MADD multiple acylCoA dehydrogenase deficiency (see main text for clinical details); NBS newborn screening; OMIM Online Mendelian Inheritance in Man (https://www.omim.org); PDHC pyruvate dehydrogenase complex; PMR psychomotor retardation 


\section{Figure legends}

\section{Figure 1a}

Dietary FAD and FMN are converted to riboflavin by hydrolases at the brush border of ileal enterocytes. Riboflavin is then absorbed into the intestine via apically expressed RFVT3 (also known as hRFT2) and released into portal blood by basolaterally expressed RFVT1 (also known as hRFT1) and RFVT2 (also known as hRFT3). Circulating plasma riboflavin is transported in blood bound to either albumin or immunoglobulins. RFVT2-mediated transport allows riboflavin uptake into the brain. The precise mechanism of import of riboflavin into the mitochondrial matrix has not been established to date. We have depicted RFVT? as a putative riboflavin transporter responsible for this step. Subsequent conversion into flavocoenzymes occurs in tissue cells, as shown in figure $1 \mathrm{~b}$. The mitochondrial FAD-transporter (FADT) is an inner mitochondrial membrane carrier that imports FAD from the cytosol into the mitochondria. The question mark indicates that FADT-mediated efflux of FAD from the mitochondrial matrix to the cytosol remains to be established. See also Barile et al, J Inher Metab Dis (2016): 39; 545-557.

\section{Figure 1b}

After cellular uptake of riboflavin, flavocoenzymes are formed by a sequential pathway involving riboflavin kinase and FAD synthase. Both steps require ATP. $\mathrm{Zn}^{2+}$ is the preferred metal ion which facilitates the ${ }^{3}$-phosphate exchange catalysed by riboflavin kinase, whilst $\mathrm{Mg}^{2+}$ is used in the adenylation catalysed by FAD synthase. 


\section{Diet}

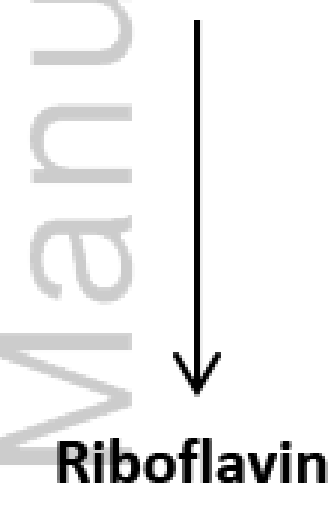

\section{Riboflavin}

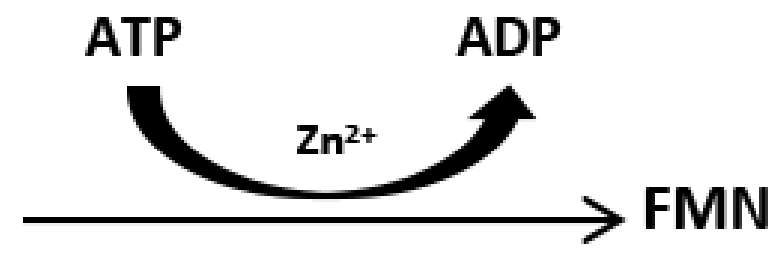

Riboflavin

kinase

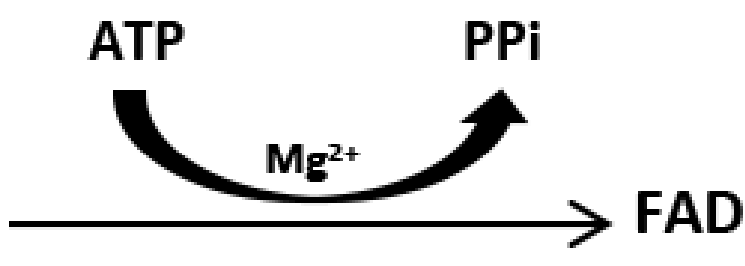

FAD

synthase

JIMD_12058_Fig 1b.tif 


\section{University Library}

\section{- M M I E E R VA A gateway to Melbourne's research publications}

Minerva Access is the Institutional Repository of The University of Melbourne

Author/s:

Balasubramaniam, S;Christodoulou, J;Rahman, S

Title:

Disorders of riboflavin metabolism

Date:

2019-07-01

Citation:

Balasubramaniam, S., Christodoulou, J. \& Rahman, S. (2019). Disorders of riboflavin metabolism. JOURNAL OF INHERITED METABOLIC DISEASE, 42 (4), pp.608-619. https:// doi.org/10.1002/jimd.12058.

Persistent Link:

http://hdl.handle.net/11343/285569 